\title{
Escape of rock-forming volatile elements and noble gases from planetary embryos
}

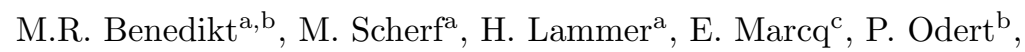 \\ M. Leitzinger ${ }^{\mathrm{b}}$, N.V. Erkaev ${ }^{\mathrm{d}, \mathrm{e}}$ \\ 5 \\ ${ }^{a}$ Space Research Institute, Austrian Academy of Sciences, Schmiedlstraße 6, 8042 Graz, \\ Austria \\ ${ }^{b}$ Institute of Physics, IGAM, University of Graz, Universitätsplatz 5, 8010 Graz, Austria \\ ${ }^{c}$ Université de Versailles St-Quentin-En-Yvelines, France \\ ${ }^{d}$ Institute of Computational Modelling of the Siberian Branch of the Russian Academy of \\ Sciences, 660036 Krasnoyarsk, Russian Federation \\ e Siberian Federal University, 660041 Krasnoyarsk, Russian Federation
}

\begin{abstract}
Large planetesimals and planetary embryos ranging from several hundred to a few thousand kilometers can develop magma oceans through mutual collisions, gravitational energy, and the heating of short-lived radioactive elements. During their solidification after the dissipation of the disk a steam atmosphere will be catastrophically outgassed and may be lost efficiently via hydrodynamic escape, as long as it does not condense. The escaping $\mathrm{H}$-atoms that originate from the dissociation of $\mathrm{H}_{2} \mathrm{O}$ and $\mathrm{H}_{2}$ will drag heavier trace elements like noble gases such 20 as $\mathrm{Ne}$ and Ar and outgassed moderately volatile rock-forming elements such as $\mathrm{K}, \mathrm{Na}, \mathrm{Si}, \mathrm{Mg}$, etc. into space. Under consideration of various EUV flux evolution scenarios of young solar-type stars, we apply an upper atmosphere hydrodynamic escape model that includes the dragging of heavier species by escaping $\mathrm{H}$-atoms. We investigate the atmospheric/elemental escape and fractionation from planetary embryos with masses of $1 M_{\text {Moon }}, 0.5 M_{\text {Mars }}, 1 M_{\text {Mars }}$, and 1.5 $M_{\text {Mars }}$ at different orbital distances between the orbits of Venus and Mars. Our results indicate that the steam atmospheres and the embedded trace elements will be lost efficiently before they condense for masses $\leq 0.5 M_{\text {Mars }}$ and orbital distances up to $1 \mathrm{AU}$. For heavier embryos of up to $1.5 M_{\text {Mars }}$ almost all of so the considered steam atmospheres can be lost within $\approx 12 \mathrm{Myr}$, which lies within the time frame of the formation of the first Martian protocrust after $\approx 20 \mathrm{Myr}$,
\end{abstract}


i.e. for such steam atmospheres to be lost completely a shallow magma ocean must remain below the atmosphere, which might be achieved through frequent impacts onto the planetary embryo. The considered outgassed noble gases and rock-forming elements will be completely dragged away together with the steam atmospheres under the assumption that the trace elements will reach the thermosphere. For embryos with masses $\leq M_{\text {Moon }}$ the gravity is too weak for a dense atmosphere to build up for the high magma ocean related surface temperatures and all outgassed elements will escape immediately to space. For all considered planetary masses and orbits the loss rates of Ar and Ne are so high that there will be no fractionation of their isotopes. The studied planetary embryos, even though not isotopically fractionated, will therefore be severely depleted in noble gases and moderately volatile elements. Hydrodynamic escape might then also affect the final composition of terrestrial planets that accrete out of such 45 planetary embryos, such as the volatile content and the $\mathrm{Fe} / \mathrm{Mg}$ ratio of a planet. Keywords: Atmospheres evolution, Solar radiation, Planetary formation

\section{Introduction}

During the early evolution of planetary systems large planetesimals and planetary embryos are formed out of dust and ice, while embedded in the circumstel50 lar disks (Wood, 2005; Birnstiel et al., 2016; Blum, 2018). Within the first 3 Myr following the birth of the solar system, 26Al, the most abundant short-lived radioactive heat source capable of melting rocky material, becomes extinct. Heating by short-lived radioisotopes such as ${ }^{60} \mathrm{Fe}$ and ${ }^{26} \mathrm{Al}$ determined the thermal history and interior structure of these planetary building blocks during the earliest stages of planetary formation (Lichtenberg et al., 2016a, 2018). The following thermo-mechanical evolution, like internal differentiation and rapid volatile outgassing, yields important implications for the final composition and hence the evolution of terrestrial planets. The accreting planetary bodies whose radii range from tens to hundreds of kilometers (Elkins-

60 Tanton, 2012; Lichtenberg et al., 2016b, 2018) and more, experienced significant 
and perhaps complete melting due to radiogenic heating from these short-lived radioisotopes (Urey, 1955; Fish et al., 1960; Lichtenberg et al., 2016b,a, 2018, 2019; Hin et al., 2017; Young et al., 2019) as well as during energetic accretionary impacts with other large planetesimals and planetary embryos (Safronov \& Zv-

65 jagina, 1969; Wetherill, 1980; Tonks \& Melosh, 1993).

The magma oceans on these planetary building blocks are responsible for differentiation and their resulting composition. During this hot magmatic phase thermodynamic models have shown that moderately volatile rock-forming elements such as $\mathrm{Na}, \mathrm{K}, \mathrm{Si}, \mathrm{Mg}, \mathrm{Fe}$, Ca, etc. are outgassed from the magmatic surface (Schaefer \& Fegley, 2007; Fegley et al., 2016; Young et al., 2019; Sossi et al., 2019). The amount of degassing volatiles from the magma ocean strongly depends on its temperature and oxygen fugacity (e.g. Sossi et al., 2019), but also on its bulk composition and solidification (Elkins-Tanton, 2008, 2012), which is interconnected with the building blocks of the planetary embryos. Even though recent studies by Hin et al. (2017) and Young et al. (2019) investigated the loss of silicates from evaporating magma oceans around low-mass planetesimals and embryos, until now no study investigated the possible escape of such elements via EUV-driven hydrodynamic drag of catastrophically outgassed steam atmosphere related H-atoms from larger planetary embryos after the disk dissipated 80 and the magma ocean solidified.

It is very likely that processes such as atmospheric escape and collisional erosion altered the bulk composition of the Earth from its initial stages of accretion, which is often assumed to be chondritic, to the presentday. Earth's Si/Mg ratio for instance may have evolved due to stepwise losses from the accreting planetary building blocks (Fegley et al., 2016), or the elevated $\mathrm{Mn} / \mathrm{Na}$ ratio of small telluric bodies relative to chondrites might reflect the oxidised conditions of a magma ocean phase since Na becomes more volatile under oxidised environments than Mn (e.g. O'Neill \& Palme, 2008; Siebert et al., 2018). The different bulk com90 positions due to losses of $\mathrm{Mg}$, Si etc. through escape of early-formed steam atmospheres alter the density, and interior structure of planetary embryos. The 
losses of $\mathrm{Na}, \mathrm{K}, \mathrm{Si}, \mathrm{Al}, \mathrm{Ca}$, from crusts may further alter the composition and structure of planetary embryos and accreting protoplanets. The abundance of the isotope ${ }^{40} \mathrm{~K}$, for instance, is important since its radioactive decay contributes to the thermal evolution of the interiors of planetary embryos and protoplanets and global processes such as the generation of long-lived magnetic dynamos (Turcotte \& Schubert, 2002; Murthy et al., 2003; Nimmo et al., 2004; Nimmo \& Kleine, 2015). In addition, elemental ratios such as $\mathrm{Fe} / \mathrm{Mg}$ and $\mathrm{Si} / \mathrm{Fe}$, which can also be altered by collisional erosion (O'Neill \& Palme, 2008; Carter et al., 2015; Bonsor et al., 2015; Boujibar et al., 2015), might also be affected by escape of an early steam atmosphere (Fegley et al., 2016). Moreover, moderately volatile elements can be fractionated from each other through their loss from planetary embryos in dependence of their equilibrium pressure (Sossi et al., 2019). In case of collisional erosion, elements will be fractionated according to their incompatibility in mantle minerals during melting, while atmospheric loss preferentially removes volatile elements.

Fig. 1 illustrates this process. Planetary embryos will experience a magma ocean phase during their accretion due to radioactive heating, gravitational energy and frequent impacts. Due to the high magma ocean temperature moderately volatile elements will outgas in dependence of the volatility and equilibrium pressure and form a silicate vapor atmosphere. After dissipation of the solar nebula this atmosphere will be susceptible to atmospheric escape, for low mass bodies due to the high surface temperature and, for more massive bodies, also due the strong EUV flux from the young Sun. While small embryos might lose these volatiles, bigger embryos might accumulate a silicate atmosphere owing to their greater gravitational pull. When the magma ocean starts to solidify a steam atmosphere - in dependence of the oxygen fugacity mainly consisting either of $\mathrm{H}_{2} \mathrm{O}$ and $\mathrm{CO}_{2}$ (oxidized magma ocean) or $\mathrm{H}_{2}$ and $\mathrm{CO}$ (reduced magma ocean) - will start to catastrophically outgas and the silicate fraction will be mixed with the outgassed volatiles. Due to the high EUV flux of the young star/Sun, $\mathrm{H}_{2} \mathrm{O}$ and $\mathrm{H}_{2}$ will be dissociated and atomic hydrogen dominates the upper atmosphere and will escape from the planetary embryo hydrodynami- 
cally. Heavier elements such as the moderately volatile rock-forming elements and noble gases - thereafter also called 'trace elements' - that reach the hydrogen dominated thermosphere will then be dragged along with the escaping hydrogen in dependence of their different masses, thus, be lost from the embryo.

Below the escaping steam atmosphere a shallow magma ocean might remain due to frequent smaller impactors which will also lead to further outgassing of moderately volatiles to keep the respective equilibrium pressures of the different escaping species. If no shallow magma ocean remains the escaping steam atmosphere will either condense or be lost completely before condensation can occur, which in turn is dependent on the orbital location. Depending on the escape rates - which are linked to the mass, surface temperature, EUV flux, and condensation time - this process will then deplete moderately volatile elements and can potentially also fractionate their isotopes and/or lighter from heavier elements. How and if this process affects depletion and fractionation of the outgassed elements on planetary embryos, however, will be the main aim of this study.

Therefore, we investigate the losses of the rock-forming elements $\mathrm{Na}, \mathrm{K}, \mathrm{Si}$, and $\mathrm{Mg}$ and of the non-radiogenic noble gas isotopes ${ }^{36} \mathrm{Ar},{ }^{38} \mathrm{Ar},{ }^{20} \mathrm{Ne},{ }^{22} \mathrm{Ne}$ from planetary embryos with $0.01 M_{\text {Earth }}\left(1.0 M_{\text {Moon }}\right), 0.05 M_{\text {Earth }}\left(0.5 M_{\text {Mars }}\right)$, $0.1 M_{\text {Earth }}\left(1.0 M_{\text {Mars }}\right)$ and $0.15 M_{\text {Earth }}\left(1.5 M_{\text {Mars }}\right)$, hereafter referred to as $M_{\mathrm{Emb}}=0.5-1.5 M_{\mathrm{Mars}}$, in orbit locations at 0.7, 1.0 and $1.5 \mathrm{AU}$ exposed to the EUV flux of a young solar-like G-star after the disk disappeared.

To study these losses, we assume

- catastrophically outgassed steam atmospheres from a solidifying magma ocean that formed on the planetary embryos due to radioactive decay, gravitational energy, and frequent impactors during their growth;

- different degassed amounts of $\mathrm{CO}_{2}$ and $\mathrm{H}_{2} \mathrm{O}$ that will subsequently be lost through hydrodynamic escape;

- degassing of $\mathrm{Na}, \mathrm{K}, \mathrm{Si}$, and $\mathrm{Mg}$, as well as ${ }^{36} \mathrm{Ar},{ }^{38} \mathrm{Ar},{ }^{20} \mathrm{Ne},{ }^{22} \mathrm{Ne}$ (the 'trace elements') into the steam atmosphere that can then be dragged 
away from the upper atmosphere by the dissociation products of $\mathrm{H}_{2} \mathrm{O}$;

- and that a shallow magma ocean remains beneath the steam atmosphere due to frequent impacts. If no shallow magma ocean remains, the steam atmosphere might in reality condense before it escapes completely, which strongly depends on the orbital location of the planetary embryo.

To calculate the losses of the moderately volatile elements and of the noble gases, we simulate

- the atmospheric structure of the different steam atmospheres from the surface up to the $1 \mu$ bar-level with a $1 \mathrm{D}$ radiative-convective atmosphere model for planetary embryos of $M_{\mathrm{Emb}}=0.5-1.5 M_{\text {Mars }}$, magma ocean surface temperatures of $\mathrm{T}_{\text {surf }}=1500-3000 \mathrm{~K}$ (Marcq, 2012; Marcq et al., 2017; Pluriel et al., 2019), and different orbital locations;

- the evolution of the steam atmosphere through energy-limited escape (e.g. Watson et al., 1981);

- and the escape of the trace elements through dragging by the hydrodynamically escaping hydrogen and oxygen atoms (Zahnle \& Kasting, 1986; Hunten et al., 1987; Odert et al., 2018).

In Section 2 magma ocean parameters and the initial abundances of the studied elements as well as outgassed atmospheric fractions of the elements are discussed. Section 3 summarizes the radiative-convective magma ocean related steam atmosphere model that is used for the calculation of the atmosphere heights. In Section 4 we briefly describe hydrodynamic escape and the upper atmosphere escape model that is used for the calculation of the escape rates of the elements at different orbital locations and the therewith connected steam atmosphere parameters. We study the losses along expected solar EUV evolution tracks that correspond to slowly to moderately rotating young solar-like G-stars (Tu et al., 2015). Section 5 presents the results and discusses the findings of the 180 study in the context of early Earth's accretion. 


\section{Planetary embryo composition and magma oceans}

As mentioned in Section 1, Moon-mass and larger planetary embryos went through magma ocean phases as a result of efficient heating due to collisions, radioactive decay and/or gravitational energy (e.g. Elkins-Tanton, 2012; Lichtenberg et al., 2018; Albarède \& Blichert-Toft, 2007). For the embryos of 0.05, 0.1 , and $0.15 M_{\text {Earth }}$ we assume magma ocean depths of $1000 \mathrm{~km}$ which is in good agreement with siderophile-element abundances and silicate partitioning data as obtained for Mars (Agee \& Draper, 2004; Righter et al., 1998; Righter \& Chabot, 2011; Elkins-Tanton, 2012). Such magma ocean depth covers $75 \%$ of

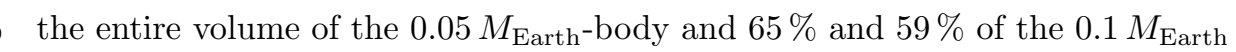
and $0.15 M_{\text {Earth-bodies, respectively. }}$

According to Elkins-Tanton (2008, 2012), Lebrun et al. (2013), Hamano et al. (2013) and Massol et al. (2016) the solidification of magma oceans is a relatively fast process and occurs within $\approx 10^{5}$ years for $1.5 \mathrm{AU}, \approx 1.5 \mathrm{Myr}$ at $1 \mathrm{AU}$, and $\geq 10 \mathrm{Myr}$ at $0.7 \mathrm{AU}$, respectively. A shallow magma ocean, however, can remain after the solidification of the deep global magma ocean. A study by Maindl et al. (2015) for instance indicates that due to frequent impacts a shallow magma ocean might have been sustained for several million years. This is also supported by a recent study of the solidification of the martian magma ocean (Bouvier et al., 2018). This investigation of the U-Pb chronology of Martian zircons indeed indicates that the first Martian protocrust formed at about $20 \mathrm{Myr}$ after the formation of the Solar System. Mars itself has accreted a mass of $0.63 M_{\text {Mars }}$ after $\approx 2.4 \mathrm{Myr}$ and completely formed after $\approx 10 \mathrm{Myr}$ (Dauphas \& Pourmand, 2011), meaning that the fully formed Mars had a protracted magma ocean for about 10 Myr. This subsequently implies that atmospheric escape can certainly play an important role in volatile depletion on a planet with a protracted magma ocean phase, since the extent of atmospheric loss on a planets volatile budget is the integral of the effective escape time.

We assume for the Moon-mass planetary embryos magma oceans depths as derived from Lunar studies. The picritic glasses, mare basalts and potassium 
Table 1: CC composition of relevant elements and their isotopes (based on Lodders (2003); Lodders et al. (2009)).

\begin{tabular}{ccc|ccc}
\hline noble gases & \multicolumn{2}{c}{ amount } & elements & \multicolumn{2}{c}{ amount } \\
& {$[\mathrm{ppm}]$} & {$[\mathrm{mol} / \mathrm{g}]$} & & {$[\mathrm{ppm}]$} & {$[\mathrm{mol} / \mathrm{g}]$} \\
\hline \hline $\mathrm{Ne}$ & $1.8 \times 10^{-4}$ & $8.92 \times 10^{-12}$ & $\mathrm{Na}$ & 4990 & $2.17 \times 10^{-4}$ \\
${ }^{20} \mathrm{Ne}$ & $1.60 \times 10^{-4}$ & $8.30 \times 10^{-12}$ & $\mathrm{Mg}$ & 95800 & $3.94 \times 10^{-3}$ \\
${ }^{22} \mathrm{Ne}$ & $1.20 \times 10^{-5}$ & $6.10 \times 10^{-13}$ & $\mathrm{Si}$ & 107000 & $3.81 \times 10^{-3}$ \\
$\mathrm{Ar}$ & $1.33 \times 10^{-3}$ & $3.33 \times 10^{-11}$ & $\mathrm{~K}$ & 544 & $1.40 \times 10^{-5}$ \\
${ }^{36} \mathrm{Ar}$ & $1.13 \times 10^{-3}$ & $2.82 \times 10^{-11}$ & $\mathrm{Fe}$ & $185 \times 10^{3}$ & $3.32 \times 10^{-3}$ \\
${ }^{38} \mathrm{Ar}$ & $2.04 \times 10^{-4}$ & $5.12 \times 10^{-12}$ & $\mathrm{U}$ & $8.10 \times 10^{-3}$ & $3.40 \times 10^{-11}$ \\
\hline
\end{tabular}

$(\mathrm{K})$, rare-Earth elements (REE) and phosphorus (P), as well as the so-called KREEP component of enriched incompatible elements, are consistent with fractional solidification of a lunar magma ocean that was originally hundreds of kilometers deep (Philpotts \& Schnetzler, 1970; Wakita \& Schmitt, 1970; Elkins215 Tanton, 2012). Furthermore, from observations of Lunar serial magmatism and of a uniform orthopyroxene, olivine melting region, it is expected that the lunar magma ocean depth was between 250 - 500 km (Elkins-Tanton et al., 2011; Schaefer \& Elkins-Tanton, 2018). By assuming a similar size and mass for the smallest planetary embryos in our study we assume comparable magma ocean depths of $500 \mathrm{~km}$ for $0.01 M_{\text {Earth }}$ (Moon-mass) embryos. We investigate the losses of the rock-forming elements from these Moon-mass embryos by assuming magma ocean surface temperatures $\left(T_{\text {Surf }}\right)$ of $1500,2000,2500$ and $3000 \mathrm{~K}$.

After the disk evaporated, depending on the accretion history and orbit location, a range of possible bulk compositions for planetary embryos and accreting proto-planets can be expected. Such bodies contain a mixed rocky bulk composition with iron metal meteorites, different water abundances, or mixtures of metallic iron, silicate rock, and unconstrained volumes of icy planetesimals and planetary embryos from beyond the ice line (e.g. Brasser, 2013; Morbidelli \& Raymond, 2016; Raymond et al., 2014). Based on studies of meteorites, 

icate composition of terrestrial planets can be expected (Elkins-Tanton, 2008, 2012; Massol et al., 2016). While we vary the outgassed water inventory in this study, we assume only a pure carbonaceous chondritic (CC) composition for all planetary embryos since these are the most volatile-rich building blocks. Even

235 though our obtained results might also be valid for other bodies such as enstatite chondrites, one has to extrapolate with care. Since enstatite chondrites for instance are more reduced than carbonaceous chondrites (e.g Schaefer \& Fegley, 2017), more reduced gases are also expected to be formed during their evaporation, which might alter our results.

Besides the rock-forming elements (Schaefer \& Fegley, 2007; Schaefer \& Fegley, 2010; Fegley et al., 2016) and noble gases that outgas from the magma ocean, $\mathrm{H}_{2} \mathrm{O}$ and $\mathrm{CO}_{2}$ molecules will degas catastrophically into dense steam atmospheres during the solidification process (Elkins-Tanton, 2008, 2012; Massol

245 et al., 2016; Salvador et al., 2017). For our simulations we assume partial pressure values of 50 bar, 100 bar and 150 bar for $\mathrm{H}_{2} \mathrm{O}$ and 5 bar, 10 bar and 30 bar for $\mathrm{CO}_{2}$, which are within an expected reasonable range of volatile contents for such bodies (Elkins-Tanton, 2008, 2012). Elemental and isotopic abundances relevant to our study are calculated via Lodders (2003); Lodders et al. (2009) and listed in Table 2. We furthermore study different cases of outgassed amounts of the trace elements, i.e. $10 \%, 50 \%$ and $100 \%$ of the initial amount within the magma ocean, as well as a continuous outgassing of the studied elements in balance with the pressure equilibria between magma ocean and atmosphere.

\section{Steam atmosphere and escape simulations}

\subsection{Magma ocean related radiative-convective atmospheres}

A $1 \mathrm{D}$ radiative-convective atmosphere model is applied to the magma ocean related steam atmosphere cases discussed in Sect. 2. The model that we use is described extensively in Marcq (2012), Marcq et al. (2017), and Pluriel et al. 
(2019), and applied in several coupled magma ocean-atmosphere evolution studet al. (1984) for $\mathrm{H}_{2} \mathrm{O}$, since we can easily reach a supercritical phase from $\mathrm{H}_{2} \mathrm{O}$. This means that in the lowermost atmospheric layers, we may have 
$P_{\mathrm{H}_{2} \mathrm{O}}>P_{\text {crit- } \mathrm{H}_{2} \mathrm{O}}$ and/or $T>T_{\text {crit- } \mathrm{H}_{2} \mathrm{O}}$. In such a case, we cannot (and do not) consider $\mathrm{H}_{2} \mathrm{O}$ as an ideal gas, so we use the values given in the aforementioned steam tables for a more accurate equation of state for

$\mathbf{H}_{2} \mathbf{O}$. The pressure and temperature profiles for selected steam atmospheres up to the lower thermosphere, i.e. up to the $1 \mu$ bar-level, are shown in Fig. 2. The EUV flux, which drives the escape of the hydrogen atoms, is absorbed above this height.

Finally, the humidity ratio $\alpha_{v}=\rho_{\mathrm{H}_{2} \mathrm{O}} / \rho_{\mathrm{CO}_{2}}$ is prescribed in the three layers as follows: vertically uniform in the dry adiabat and isothermal layer, and decreasing with increasing height in the moist adiabat layer according to the expression given by Kasting (1988).

\subsection{Radiative modelling}

Rayleigh scattering is considered for both $\mathrm{H}_{2} \mathrm{O}$ and $\mathrm{CO}_{2}$ following a simple $\lambda^{-4}$ spectral dependency (Kopparapu et al., 2013; Sneep \& Ubachs, 2005). Gaseous absorption includes spectral lines from HITRAN 2012 (Turbet et al., 2017 ) as well as continuum opacities for $\mathrm{CO}_{2}-\mathrm{CO}_{2}$ (Stefani et al., 2013), $\mathrm{H}_{2} \mathrm{O}$ $\mathrm{H}_{2} \mathrm{O}$ (Mlawer et al., 2012) and $\mathrm{CO}_{2}-\mathrm{H}_{2} \mathrm{O}$ (Turbet et al., 2017). These absorption spectra are then used to compute a look-up table of $16 k$-coefficients for a grid in $\left(\alpha_{v}, T, P\right)$ using KSPECTRUM (Eymet et al., 2009), to be used in a $k$-correlated code. The model then solves the radiative transfer equation using the standard DISORT solver in the 4-stream approximation.

\subsection{Steam atmospheres $\mathcal{E}$ hydrodynamic escape}

On present Earth, Venus or Mars, hydrogen escape is limited by the upward diffusion of hydrogen through the upper atmosphere (e.g. Hunten, 1975). The diffusion-limited flux is proportional to the total hydrogen-mixing ratio at the homopause. However, the present $\mathrm{H}_{2} \mathrm{O}$ mixing ratio at the homopause level of these planets is quite low. In present-day Mars' case, for instance, the $\mathrm{H}_{2} \mathrm{O}$ mixing ratio at the homopause level is $(3 \pm 1) \times 10^{-5}$ (e.g. Krasnopolsky \& Feldman, 2001). As it was shown by Kasting \& Pollack (1983) when they 
studied the loss of water from Venus, which has an even lower $\mathrm{H}_{2} \mathrm{O}$ mixing ratio at the homopause level of $\approx 5 \times 10^{-7}$, for levels of about $\geq 5 \times 10^{-4}$ the solar EUV energy that is absorbed at higher altitudes becomes larger than the energy that is carried away by the Jeans escape flux. Depending on the orbital location of a planetary body, its mass and the host star's energy input into the upper atmosphere from the EUV range, the atmosphere can experience classical Jeans escape or hydrodynamic outflow, which can even result in blow-off (e.g. Parker, 1963; Chamberlain, 1963; Öpik, 1963; Watson et al., 1981; Bauer \& Lammer, 2004; Tian et al., 2008a,b; Erkaev et al., 2013; Lammer et al., 2013).

Jeans escape is valid as long as the upper atmosphere remains in hydrostatic conditions so that particles that populate the high-energy tail of a Maxwellian distribution at the exobase level, where the mean free path equals the scale 330 height, overcome the escape energy and are lost from the gravitational potential of the planetary body. For $\mathrm{H}_{2} \mathrm{O}$ mixing ratios $>5 \times 10^{-4}$ the upper atmosphere becomes unstable against expansion and the Jeans escape formula is not valid anymore (Kasting \& Pollack, 1983). These authors found that if the mixing ratios reach values that are $\geq 10^{-3}$ then the hydrogen escape flux reaches values that are comparable with the EUV-restricted energy-limited escape as discussed by Watson et al. (1981). These conditions are also fulfilled for the studied steam atmospheres where we have intial $\mathrm{H}_{2} \mathrm{O}$ mixing ratios at the homopause levels that are more or less $>0.1$.

In case a planetary embryo underwent a magma ocean scenario so that the body outgassed an $\mathrm{H}_{2} \mathrm{O}$-vapor-dominated steam atmosphere during its solidification, $\mathrm{H}$ atoms will be the dominant species in the upper atmosphere (e.g. Kasting \& Pollack, 1983; Chassefière, 1996b,a; Odert et al., 2018; Lammer et al., 2013; Guo, 2019). In the upper atmosphere the solar/stellar UV- and EUV radiation dissociates $\mathrm{H}_{2} \mathrm{O}$ molecules and produces $\mathrm{H}$ atoms via the reac345 tions $\mathrm{H}_{2} \mathrm{O}+\mathrm{h} \nu \rightarrow \mathrm{OH}+\mathrm{H}$ and $\mathrm{OH}+\mathrm{H}_{2} \rightarrow \mathrm{H}_{2} \mathrm{O}+\mathrm{H}$. The decomposition of $\mathrm{H}_{2} \mathrm{O}$ through these reactions is the dominant process (Huebner \& Mukherjee, 2015; Guo, 2019). If the $\mathrm{H}_{2} \mathrm{O}$-vapour mixing ratio near the homopause level remains more or less constant for different EUV and UV fluxes, then less 
$\mathrm{H}_{2} \mathrm{O}$-vapour will populate the altitudes above for high radiation fluxes due to

$\mathrm{H}_{2}$ number density above the dissociation level is one order of magnitude lower compared to atomic hydrogen (Lammer et al., 2013).

Recently, Guo (2019) studied the effect of photoionization and the loss of $\mathrm{H}_{2} \mathrm{O}$ from steam atmospheres of Earth-like planets that were exposed to EUV fluxes between 10 and 400 and FUV flux values between 5 and 200 times the present-day solar values with a hydrodynamic model that included photochemistry. It was found that in all cases the water molecules dissociate,around the $1 \mu$ bar atmospheric pressure level so that atomic $\mathbf{H}$ becomes the predominant species, while $\mathbf{O}$ atoms are the second most abundant (see Fig. 1; Guo, 2019). IR-cooling molecules such as $\mathrm{CO}_{2}$ do not reach these altitudes largely due to its weight, therefore the EUV flux is absorbed in a hydrogen-dominated upper atmosphere. The hydrogen escape rate depends on the magnitude of the stellar/solar EUV flux that drives the upper atmosphere heating and expansion and as long as hydrogen is the main species to a lesser extent, on the oxygen and $\mathrm{CO}_{2}$ content of the atmosphere (Kasting \& Pollack, 1983; Guo, 2019).

In all steam atmosphere cases that are considered in our study the solar EUV flux is absorbed above the $1 \mu$ bar level, where it deposits its energy that drives the expansion of the upper atmosphere dynamically accompanied by adiabatic cooling, so that the exobase location reaches very large distances (Watson et al., 1981; Tian et al., 2005, 2008a,b; Erkaev et al., 2013, 2014, 2015; Lammer et al., 2013), which results in an efficient escape of the hydrogen inventory or even the loss of heavy species that are outgassed from the magma ocean and dragged away by the escaping hydrogen atoms (Zahnle \& Kasting, 1986; Hunten et al., 1987; Pepin, 2006; Odert et al., 2018; Guo, 2019) if they reach the hydrogendominated region.

The Tables 2, 3, and 4 show the $1 \mu$ bar levels above the embryo surface for masses of $0.5,1.0$, and $1.5 M_{\text {Mars }}$ for different surface temperatures of 1500$3000 \mathrm{~K}$ and orbital locations from 0.5-1.5 AU calculated with the 1 D-radiative atmosphere model for the considered planetary embryo masses, surface tem- 
Table 2: Input parameters and resulting atmospheric heights above the surface used for $M_{\text {Emb }}=0.5 M_{\text {Mars }}$.

\begin{tabular}{|c|c|c|c|c|c|}
\hline Cases & $\mathrm{d}[\mathrm{AU}]$ & $T_{\text {surf }}[\mathrm{K}]$ & $\begin{array}{l}50 \text { bar } \mathrm{H}_{2} \mathrm{O} \\
5 \text { bar } \mathrm{CO}_{2}\end{array}$ & $\begin{array}{c}100 \text { bar } \mathrm{H}_{2} \mathrm{O} \\
10 \text { bar } \mathrm{CO}_{2}\end{array}$ & $\begin{array}{c}150 \text { bar } \mathrm{H}_{2} \mathrm{O} \\
30 \text { bar } \mathrm{CO}_{2}\end{array}$ \\
\hline & & & \multicolumn{3}{|c|}{$1 \mu$ bar level $[\mathrm{km}]$} \\
\hline \multirow{4}{*}{ I } & \multirow{4}{*}{0.5} & 1500 & 2210 & 2292 & 1998 \\
\hline & & 2000 & 3806 & 3943 & 3313 \\
\hline & & 2500 & 7541 & 7806 & 6060 \\
\hline & & 3000 & 24386 & 26608 & 14886 \\
\hline \multirow{4}{*}{ II } & \multirow{4}{*}{0.7} & 1500 & 2210 & 2292 & 1998 \\
\hline & & 2000 & 3806 & 3943 & 3313 \\
\hline & & 2500 & 7541 & 7806 & 6060 \\
\hline & & 3000 & 24386 & 26608 & 14886 \\
\hline \multirow{4}{*}{ III } & \multirow{4}{*}{1} & 1500 & 2210 & 2292 & 1998 \\
\hline & & 2000 & 3806 & 3943 & 3313 \\
\hline & & 2500 & 7541 & 7806 & 6060 \\
\hline & & 3000 & 24386 & 26608 & 14886 \\
\hline \multirow{4}{*}{ IV } & \multirow{4}{*}{1.5} & 1500 & 2210 & 2292 & 1998 \\
\hline & & 2000 & 3806 & 3943 & 3313 \\
\hline & & 2500 & 7541 & 7806 & 6060 \\
\hline & & 3000 & 24386 & 26608 & 14886 \\
\hline
\end{tabular}


Table 3: Input parameters and resulting atmospheric heights above the surface used for $M_{\mathrm{Emb}}=1.0 M_{\mathrm{Mars}}$

\begin{tabular}{|c|c|c|c|c|c|}
\hline Cases & $\mathrm{d}[\mathrm{AU}]$ & $\mathrm{T}_{\text {surf }}[\mathrm{K}]$ & $\begin{array}{l}50 \text { bar } \mathrm{H}_{2} \mathrm{O} \\
5 \text { bar } \mathrm{CO}_{2}\end{array}$ & $\begin{array}{c}100 \text { bar } \mathrm{H}_{2} \mathrm{O} \\
10 \text { bar } \mathrm{CO}_{2} \\
1 \mu \text { bar level }[\mathrm{km}\end{array}$ & $\begin{array}{l}150 \text { bar } \mathrm{H}_{2} \mathrm{O} \\
30 \text { bar } \mathrm{CO}_{2}\end{array}$ \\
\hline \multirow{4}{*}{ V } & \multirow{4}{*}{0.5} & 1500 & 1347 & 1385 & 1245 \\
\hline & & 2000 & 1986 & 2032 & 1809 \\
\hline & & 2500 & 2946 & 3008 & 2629 \\
\hline & & 3000 & 4477 & 4567 & 3896 \\
\hline \multirow{4}{*}{ VI } & \multirow{4}{*}{0.7} & 1500 & 1347 & 1385 & 1245 \\
\hline & & 2000 & 1986 & 2032 & 1809 \\
\hline & & 2500 & 2946 & 3008 & 2629 \\
\hline & & 3000 & 4477 & 4567 & 3896 \\
\hline \multirow{4}{*}{ VII } & \multirow{4}{*}{1} & 1500 & 1347 & 1385 & 1245 \\
\hline & & 2000 & 1986 & 2032 & 1809 \\
\hline & & 2500 & 2946 & 3008 & 2629 \\
\hline & & 3000 & 4477 & 4567 & 3896 \\
\hline \multirow{4}{*}{ VIII } & \multirow{4}{*}{1.5} & 1500 & 1347 & 1385 & 1245 \\
\hline & & 2000 & 1986 & 2032 & 1809 \\
\hline & & 2500 & 2946 & 3008 & 2629 \\
\hline & & 3000 & 4477 & 4567 & 3896 \\
\hline
\end{tabular}


Table 4: Input parameters and resulting atmospheric heights above the surface used for $M_{\mathrm{Emb}}=1.5 M_{\mathrm{Mars}}$

\begin{tabular}{|c|c|c|c|c|c|}
\hline Cases & $\mathrm{d}[\mathrm{AU}]$ & $\mathrm{T}_{\text {surf }}[\mathrm{K}]$ & $\begin{array}{l}50 \text { bar } \mathrm{H}_{2} \mathrm{O} \\
5 \text { bar } \mathrm{CO}_{2}\end{array}$ & $\begin{array}{c}100 \text { bar } \mathrm{H}_{2} \mathrm{O} \\
10 \text { bar } \mathrm{CO}_{2} \\
1 \mu \text { bar level }[\mathrm{km}\end{array}$ & $\begin{array}{l}150 \text { bar } \mathrm{H}_{2} \mathrm{O} \\
30 \text { bar } \mathrm{CO}_{2}\end{array}$ \\
\hline \multirow{4}{*}{ IX } & \multirow{4}{*}{0.5} & 1500 & 1075 & 1103 & 1001 \\
\hline & & 2000 & 1524 & 1555 & 1403 \\
\hline & & 2500 & 2136 & 2172 & 1941 \\
\hline & & 3000 & 3982 & 3027 & 2677 \\
\hline \multirow{4}{*}{$\mathrm{X}$} & \multirow{4}{*}{0.7} & 1500 & 1075 & 1103 & 1001 \\
\hline & & 2000 & 1524 & 1555 & 1403 \\
\hline & & 2500 & 2136 & 2172 & 1941 \\
\hline & & 3000 & 3982 & 3027 & 2677 \\
\hline \multirow{4}{*}{ XI } & \multirow{4}{*}{1} & 1500 & 1075 & 1103 & 1001 \\
\hline & & 2000 & 1524 & 1555 & 1403 \\
\hline & & 2500 & 2136 & 2172 & 1941 \\
\hline & & 3000 & 3982 & 3027 & 2677 \\
\hline \multirow{4}{*}{ XII } & \multirow{4}{*}{1.5} & 1500 & 1075 & 1103 & 1001 \\
\hline & & 2000 & 1524 & 1555 & 1403 \\
\hline & & 2500 & 2136 & 2172 & 1941 \\
\hline & & 3000 & 3982 & 3027 & 2677 \\
\hline
\end{tabular}


peratures, and steam atmosphere partial pressures. For planetary embryos of 0.1 $M_{\text {Mars }}$, however, no table is given because these hot and low-mass bodies cannot build up a stable steam atmosphere due to the low gravity and high surface temperatures which lead to extreme losses of the volatiles. This process can be compared with the so-called 'boil-off' escape that occurs at very close-in and hot sub-Neptune-type exoplanets (e.g. Fossati et al., 2017; Owen \& Wu, 2016; Lammer et al., 2016).

As discussed before, the catastrophically outgassed steam atmospheres of the planetary embryos escape hydrodynamically powered by the high EUV flux of the young star/Sun (Tu et al., 2015; Erkaev et al., 2015; Odert et al., 2018). For the escape calculations of the hydrodynamic H-atom escape flux with atomic $\mathrm{O}$ (from dissociated $\mathrm{H}_{2} \mathrm{O}$ molecules) as the heavy major component and the heavy minor trace species $\left({ }^{36} \mathrm{Ar},{ }^{38} \mathrm{Ar},{ }^{20} \mathrm{Ne},{ }^{22} \mathrm{Ne}, \mathrm{CO}_{2}, \mathrm{Na}, \mathrm{K}, \mathrm{Si}, \mathrm{Mg}\right)$ that are embedded in the outflow, we apply the same escape model and equations based on the energy-limited approach (Watson et al., 1981; Zahnle \& Kasting, 1986; Hunten et al., 1987; Erkaev et al., 2016; Odert et al., 2018). Here, we assume that the outgassed rock-forming elements are able to reach the upper thermosphere, making our estimates likely a maximum. Shock waves and a turbulent mixing of the atmosphere, by impacting material for instance, might be responsible to lift up these elements (e.g. Genda \& Abe, 2003; TrigoRodríguez \& Martín-Torres, 2013).

Since we aim to study the loss of $\mathrm{H}$ atoms that drag away $\mathrm{O}$ atoms, $\mathrm{CO}_{2}$ molecules and the above mentioned embedded trace elements, we apply a formalism for the calculation of the fractionation factors, which is applicable to more than two species. To obtain the escape rates of the above mentioned trace elements, we follow Odert et al. (2018) and use the method of Zahnle \& Kasting (1986) and Zahnle et al. (1990). We apply the improved analytical solutions given in Zahnle \& Kasting (1986) and Zahnle et al. (1990) of the general hydrodynamic multi-component equations (Hunten et al., 1987). These equations are applicable to atmospheres with one or two major species and an arbitrary amount of additional trace species (Zahnle \& Kasting, 1986; Odert 
et al., 2018). For the fractionation factors of the various trace species, we apply the analytical approximations given in Zahnle et al. (1990) that are based on simplifications such as subsonic flow and isothermal conditions of the multispecies hydrodynamic equations. These equations, however, provide also good approximations for transonic atmospheric escape, since the relative fluxes of the species are already determined in the subsonic region (Zahnle et al., 1990). It should be noted that the trace elements feel the drag of both main constituents, i.e. $\mathbf{O}$ and $\mathbf{H}$, but they themselves cannot influence the bulk flow of these species or other trace elements due to their low abundance.

The hydrogen escape flux $F_{\mathrm{H}}$ is described for a multi-component atmosphere based on the energy-limited approach by (Zahnle \& Kasting, 1986; Hunten et al., 1987; Zahnle et al., 1990; Odert et al., 2018), i.e.

$$
F_{\mathrm{H}}=\frac{\beta^{2} \eta F_{\mathrm{EUV}}}{4 \Delta \Phi\left(m_{\mathrm{H}}+m_{\mathrm{O}} f_{\mathrm{O}} x_{\mathrm{O}}+\sum_{i=1}^{n} m_{\mathrm{i}} f_{\mathrm{i}} x_{\mathrm{i}}\right)} .
$$

F Here, $m_{\mathrm{H}}$ is the mass of hydrogen, $m_{\mathrm{O}}$ of oxygen, $m_{i}$ of the trace elements (that is, the rock-forming elements and the Ar and Ne isotopes in this case), $\eta \approx 15 \%$ (Shematovich et al., 2014) is the heating efficiency in a hydrogen dominated atmosphere, $F_{\mathrm{EUV}}$ is the stellar EUV flux at the orbit location, $\Delta \Phi$ is the gravitational potential at the surface of the planetary embryo. We define $\beta$ as the ratio of the atmospheric planetocentric radius at the $1 \mu b a r$ level, the distance where the $\mathrm{H}_{2} \mathrm{O}$ molecules are dissociated and atomic hydrogen becomes the dominant species, to the embryo surface radius. The $1 \mu b a r$ level, which is illustrated in Fig. 3, hence constitutes the upper atmospheric boundary in our simulation.

The mixing ratios $\mathrm{f}_{\mathrm{i}}$ and the corresponding fractionation factors $\mathrm{x}_{\mathrm{O}}$ and $\mathrm{x}_{\mathrm{i}}$ are time dependent and can be written after Zahnle et al. (1990) and Odert 435 et al. (2018)

$$
x_{\mathrm{O}}=1-\frac{g\left(\Delta m_{\mathrm{O}, \mathrm{H}}\right) b_{\mathrm{H}, \mathrm{O}}}{F_{\mathrm{H}} k_{\mathrm{B}} T\left(1+f_{\mathrm{O}}\right)},
$$


and

$$
x_{\mathrm{i}}=\frac{1-\frac{g\left(\Delta m_{\mathrm{i}, \mathrm{H}}\right) b_{\mathrm{H}, \mathrm{i}}}{F_{\mathrm{H}} k_{\mathrm{B}} T}+\frac{b_{\mathrm{H}, \mathrm{i}}}{b_{\mathrm{H}, \mathrm{O}}} f_{\mathrm{O}}\left(1-x_{\mathrm{O}}\right)+\frac{b_{\mathrm{H}, \mathrm{i}}}{b_{\mathrm{O}, \mathrm{i}}} f_{\mathrm{O}} x_{\mathrm{O}}}{1+\frac{b_{\mathrm{H}, \mathrm{i}}}{b_{\mathrm{O}, \mathrm{i}}} f_{\mathrm{O}}},
$$

with $\Delta m_{\mathrm{O}, \mathrm{H}}$ and $\Delta m_{\mathrm{i}, \mathrm{H}}$ the mass difference between the heavy species and $\mathrm{H}$ atoms, $g$ is the gravitational acceleration at the base of the flow, i.e. the $1 \mu$ bar level, $k_{\mathrm{B}}$ is the Boltzmann constant, $T$ the upper atmosphere temperature (not to be confused with $\mathbf{T}_{\text {surf }}$ from Tables 2 and 3 ). We use the binary diffusion coefficients of $\mathrm{O}, \mathrm{CO}_{2}, \mathrm{Ar}$, Ne within hydrogen and oxygen from Zahnle $\&$ Kasting (1986). For the other trace species $i$ the binary diffusion coefficients $b$ are derived from the Chapman-Enskog approximation (Chapman and Cowling, 1970; Mason and Marrero, 1970) and can be written as

$$
b_{j, i} \approx C \sqrt{\frac{T}{\mu}}
$$

where the index $j$ corresponds to the main species $\mathrm{H}$ and later $\mathrm{O}$, while $i$ corresponds to the trace species and $\mu_{j, i}$ is the reduced mass of the involved particles. The factor $C$ is $\approx 1.52 \times 10^{18}$ and related to the reciprocal value of the hard sphere collision cross section (Hunten, 1973). Because $\mu_{j, i}=m_{j} m_{i} /\left(m_{j}+m_{i}\right)$, the binary diffusion coefficients $b_{\mathrm{j}, \mathrm{i}}$ in units of $\mathrm{cm}^{-1} \mathrm{~s}^{-1}$ for the other trace species $i$ within hydrogen and oxygen can then be estimated from the relation given in Koskinen et al. (2013), i.e.

$$
b_{\mathrm{j}, \mathrm{i}}=1.52 \times 10^{18} \sqrt{\frac{1}{m_{\mathrm{j}}}+\frac{1}{m_{\mathrm{i}}}} T^{0.5} .
$$

A detailed analysis of the EUV-evolution tracks of young Sun-like stars was carried out by Tu et al. (2015). For stars younger than about 2 Gyr, the possible range of rotation related high-energy emission is large. Here we investigate the atmospheric escape for so-called slow and moderate rotators or weakly and moderately active young G-stars along their EUV evolution tracks. As discussed in Tu et al. (2015), at very young ages the EUV emission is saturated at a roughly constant fraction of the star's bolometric luminosity $L_{\mathrm{EUV}} \sim 10^{-3} L_{\mathrm{bol}}$ (Christian \& Athioudakis, 2002), for $\approx 5 \mathrm{Myr}, 25 \mathrm{Myr}$ and $225 \mathrm{Myr}$, for slow, moderate, and fast rotator tracks. We therefore do not consider fast rotators in 
this study since the $\mathrm{H}_{2} \mathrm{O}$ contents of the magma ocean related catastrophically outgassed steam atmospheres are mainly lost within the saturation phase of a moderately rotating young G-type star (Odert et al., 2018).

The EUV flux $F_{\mathrm{EUV}}$ can be written as $F_{\mathrm{EUV}}=L_{\mathrm{EUV}} /\left(4 \pi d^{2}\right)$ with orbital distances $d$ of $0.5 \mathrm{AU}, 0.7 \mathrm{AU}, 1.0 \mathrm{AU}, 1.5 \mathrm{AU}$. We calculate the EUV-driven hydrodynamic atmospheric mass loss rate over time along slow and moderate rotator EUV luminosity evolution tracks, i.e. $L_{\mathrm{EUV}, \mathrm{s}}$ and $L_{\mathrm{EUV}, \mathrm{m}}$, respectively, based on the rotation rate distribution of young solar-like stars according to Tu et al. (2015), i.e.

$$
\begin{gathered}
L_{\mathrm{EUV}, \mathrm{s}}=5.75 \times 10^{31} t^{-0.93} \\
L_{\mathrm{EUV}, \mathrm{m}}=4.70 \times 10^{32} t^{-1.18},
\end{gathered}
$$

where $t$ is the stellar age in Myr and $L_{\mathrm{EUV}}$ the EUV luminosity in $\mathrm{erg} / \mathrm{s}$. The EUV flux, the mixing ratios and the fractionation factors are therefore calculated as functions of time. This provides the description of the evolving elemental masses for the considered isotope and elemental ratios.

Recently the solar nebula life time was estimated to be around 3.3-4.5 Myr constrained by meteorite paleomagnetism (Wang et al., 2017) and inferred from $\mathrm{Pb}$ isotopic ages of chondrules (Bollard et al., 2017). Further, the solidification of the deep magma ocean together with the catastrophically outgassing of the steam atmosphere is assumed to take place within a few hundred thousand years (e.g. Elkins-Tanton, 2008, 2012). Therefore $\approx 5 \mathrm{Myr}$ seems to be a reasonable starting time.

In the following section we present the results of the atmospheric escape model and discuss the implications of the dragged and lost rock-forming elements from dissociated planetary embryos for terrestrial planet evolution. 


\section{Results}

485

4.1. Planetary embryos between $0.5 M_{\text {Mars }}$ and $1.5 M_{\text {Mars }}$

The loss and evolution of steam atmospheres from planetary embryos at $1 \mathrm{AU}$ for a slowly rotating young G-star (cases III, VII and XI as described in Tables 2, 3 and 4 ) for $M_{\mathrm{Emb}}=0.5,1.0$ and $1.5 M_{\text {Mars }}$ and for a surface temperature of $1500 \mathrm{~K}$ is shown in Fig. 4. Even though at $1 \mathrm{AU}$ the condensation time of a 490 magma ocean is estimated to be around 1.5 Myr (see (e.g. Lebrun et al., 2013; Massol et al., 2016), here, it is assumed that a shallow magma ocean remains after the solidification of their deep global magma ocean (see also Section 2). If no shallow magma ocean can be sustained, however, the hydrodynamic escape will be reduced or even stop after the steam atmosphere condensed. In general, for scenarios in which the steam atmosphere is lost within $\approx 10 \mathrm{Myr}$ at $0.7 \mathrm{AU}$, $\approx 1.5 \mathrm{Myr}$ at $1 \mathrm{AU}$, and $\approx 0.1 \mathrm{Myr}$ at $1.5 \mathrm{AU}$ no shallow magma ocean will be needed due to the condensation time (Lebrun et al., 2013) being longer than the duration of the total escape of the steam atmosphere.

One can see in Fig. 4 that the steam atmospheres for these embryos are 500 lost within $\approx 0.9 \mathrm{Myr}$ (i.e. $5.9 \mathrm{Myr}$ after formation of the Solar System if one assumes that escape starts after $5 \mathrm{Myr}$ ) for $0.5 M_{\mathrm{Mars}} \approx 5.5 \mathrm{Myr}$ for $1.0 M_{\mathrm{Mars}}$, and within $\approx 16.7 \mathrm{Myr}$ for $1.5 \mathrm{M}_{\mathrm{Mars}}$ respectively. For cases with masses $M<$ $1.5 M_{\text {Mars }}$ even $\mathrm{CO}_{2}$ molecules will be completely lost via the hydrodynamic drag of hydrogen which is in agreement with previous studies (Erkaev et al., 2014; Odert et al., 2018). For the particular case with $M=1.5 M_{\text {Mars }}$ in Fig. 4 a small amount of $\mathrm{CO}_{2}$ and $\mathrm{O}$ might remain, but will likely be rapidly lost due to other escape processes such as subsequent thermal escape of $\mathrm{C}$ and $\mathrm{O}$ (e.g. Tian et al., 2009) or ion-escape (e.g. Dong et al., 2018).

It has to be noted that for moderate and fast rotating young solar-like stars but also for hotter surface temperatures, the atmospheres are lost even more rapidly as can be seen in Fig. 5. Here, this Figure illustrates the time of the escape of the whole atmosphere of planetary embryos with $M_{\mathrm{Emb}}=0.5,1.0$ and 1.5 $M_{\text {Mars }}$ in dependence of their orbit and surface temperature for a slow 
and moderate rotating young G-star. As one can see for $0.5 M_{\mathrm{Mars}}$ in $1 \mathrm{AU}$ most of the steam atmosphere will be lost within the formation time of the Martian crust; in general, if one assumes similar formation times for the first protocrust of planetary embryos as for Mars (Bouvier et al., 2018) then almost all of these atmospheres can be lost even for a protoplanetary mass of $1.0 M_{\mathrm{Mars}}$ up $\approx 1.5 \mathrm{AU}$. For $1.5 \mathrm{M}_{\mathrm{Mars}}$ only for a moderate rotator most of the steam atmospheres can be lost within this time frame. Additionally, one should also note that also for more reduced conditions, i.e. a magma ocean which is not oxidized and, therefore, preferentially outgasses $\mathrm{H}_{2}$ and $\mathrm{CO}$, atmospheres might be lost more rapidly than in our simulations (Odert et al., 2018).

Figures 6,7 , and 8 show the evolution of the ratios of several rock-forming elements and noble gases for the total abundance of the respective planetary embryo (i.e. total abundance within the atmosphere, magma ocean and restmass of the embryo) as mentioned above (see Table 2 for the same cases as in Fig. 4 but for all surface temperatures). These figures correspond to cases in which we assumed $100 \%$ of the trace elements to be in the steam atmosphere. Our model approach showed that, regardless of the initial value, almost the whole amount of the trace elements within the escaping steam atmosphere will be lost in any cases. The results will furthermore be nearly identical regardless whether we assume continuous outgassing from the magma ocean or everything to be outgassed at the beginning.

For the noble gases, Figures 6, 7, and 8 also display the evolution of their individual abundances (in mol/g). It can be clearly seen that all (moderately) volatile elements that will be outgassed from the magma ocean and further transported to the upper thermosphere will be completely lost together with the steam atmosphere. This also means that at such small-mass bodies noble gas isotope ratios will not be significantly fractionated by this escape process but can only be depleted. Volatile rock-forming elements and noble gases, however, will be not only depleted but also fractionated against less volatile but also against refractory elements that stay in the melt such as $\mathrm{U}$. These elements might further also be fractionated against the heavier and siderophile element 

iron resides in the core. For an undifferentiated body, however, it has to be taken into account that Fe can under certain conditions even become more volatile than $\mathrm{Mg}$ and hence also escape from the planetesimal. For $M_{\mathrm{Emb}} \leq 0.5 M_{\mathrm{Mars}}$ and $T_{\text {surf }} \geq 2500 \mathrm{~K}$ the escape related depletion occurs within $\leq 0.2 \mathrm{Myr}$. Within volatile elements even in the event that its magma ocean crystallises completely over a timescale shorter than that for atmospheric loss.

\subsection{Moon-mass and smaller planetary embryos}

For Moon-mass and smaller bodies a more simple way of estimating the escape parameters, i.e.

$$
\lambda=\frac{\mathrm{G} M_{\mathrm{emb}} m_{i}}{k_{B} T_{\mathrm{surf}} R_{\mathrm{emb}}}
$$

for the different elements on the surface of a Moon-mass embryo can be found in Table 5. Here, $\mathrm{G}$ is the Newtonian gravitational constant, $k_{B}$ the Boltzmannconstant, $M_{\mathrm{emb}}$ and $R_{\mathrm{emb}}$ are the mass and radius of the planetary embryo, and (2011) and Erkaev et al. (2015) the thermal escape regime changes over a narrow range of the critical escape parameter $\lambda_{c r i t}$; the escape is purely hydrodynamic for $\lambda_{\text {crit }} \leq 2-3$ whereas for $\lambda \geq 6$ it is not. We therefore assume that for $\lambda \leq 6$ any outgassed elements are lost immediately to space. As one can see 570 from this table, for $T_{\text {surf }} \geq 2500$ all elements studied except Fe are below $\lambda=6$; for cooler magma ocean surface temperatures some of them are slightly above. In such a case, however, one can expect that these elements nevertheless experience high escape and will be ionized and picked-up by the solar wind. 
Table 5: Escape parameters $\lambda$ on the surface of a Moon-like planetary embryo for different rock-forming elements, iron, noble gas isotopes and for $\mathrm{H}_{2} \mathrm{O}$ and its dissociation products as a function of the magma ocean surface temperature $T_{\text {surf }}$.

\begin{tabular}{ccccc}
\hline element & $\lambda(1500 \mathrm{~K})$ & $\lambda(2000 \mathrm{~K})$ & $\lambda(2500 \mathrm{~K})$ & $\lambda(3000 \mathrm{~K})$ \\
\hline \hline $\mathrm{Na}$ & 5.20 & 3.90 & 3.12 & 2.60 \\
$\mathrm{Mg}$ & 5.50 & 4.13 & 3.30 & 2.75 \\
$\mathrm{Si}$ & 6.36 & 4.77 & 3.82 & 3.18 \\
$\mathrm{SiO}$ & 9.96 & 7.47 & 5.98 & 4.98 \\
$\mathrm{~K}$ & 8.85 & 6.64 & 5.31 & 4.43 \\
$\mathrm{Fe}$ & 12.64 & 9.48 & 7.59 & 6.32 \\
$\mathrm{FeO}$ & 16.27 & 12.20 & 9.76 & 8.13 \\
${ }^{36} \mathrm{Ar}$ & 8.15 & 6.11 & 4.89 & 4.08 \\
${ }^{38} \mathrm{Ar}$ & 8.60 & 6.45 & 5.16 & 4.30 \\
${ }^{20} \mathrm{Ne}$ & 4.53 & 3.4 & 2.26 & 2.26 \\
${ }^{22} \mathrm{Ne}$ & 4.98 & 3.74 & 2.99 & 2.49 \\
\hline $\mathrm{H}_{2}$ & 0.46 & 0.34 & 0.27 & 0.23 \\
$\mathrm{O}$ & 3.60 & 2.7 & 2.17 & 1.80 \\
$\mathrm{H}_{2} \mathrm{O}$ & 4.10 & 3.10 & 2.45 & 2.09 \\
\hline
\end{tabular}

These bodies will therefore be strongly depleted in rock-forming elements and noble gases. Table 6 shows as an example of the maximum amount of elements that can be lost from an assumed $500 \mathrm{~km}$ deep magma ocean which is composed out of CC-like material as shown in Table 2. In case all of these elements in the magma ocean outgas into space then the amount given in Table 6 represents the depletion of the particular elements.

As has been shown by several different studies (e.g. Kazenas et al., 1985; ?; ?; ?) Si will most probably outgas as a molecule, i.e. SiO. Table 5 therefore also shows $\lambda$ for $\mathrm{SiO}$. For steam atmospheres, one might also consider that these elements may also form hydroxides, chlorides or sulfates, thereby increasing their molar mass and hence their $\lambda$. 
Table 6: Amount of rock-forming elements and noble gases in a $500 \mathrm{~km}$ deep magma ocean of a moon-like planetary embryo with $100 \%$ CC composition (see also Lodders et al. (2009)).

\begin{tabular}{ccc}
\hline element & amount in magma ocean $[\mathrm{g}]$ & amount in magma ocean $[\%]$ \\
\hline \hline $\mathrm{Na}$ & $1.02 \times 10^{22}$ & 0.499 \\
$\mathrm{Mg}$ & $1.85 \times 10^{23}$ & 9.58 \\
$\mathrm{Si}$ & $1.79 \times 10^{23}$ & 10.7 \\
$\mathrm{Ne}$ & $4.18 \times 10^{14}$ & $1.08 \times 10^{-8}$ \\
$\mathrm{Ar}$ & $1.56 \times 10^{15}$ & $1.33 \times 10^{-7}$ \\
$\mathrm{~K}$ & $6.53 \times 10^{20}$ & 0.0544 \\
\hline
\end{tabular}

For temperatures of $T_{\text {surf }}<2500 \mathrm{~K} \mathrm{SiO}$ would have a $\lambda$ that would be above the critical value and might therefore build up a tenuous silicate atmosphere as a consequence of an equilibrium between outgassing and escape. Such molecules, however, are also susceptible to dissociation due to the strong EUV flux. In case that the outgassing flux from the magma ocean cannot be maintained this atmosphere will soon be depleted by various escape processes such as ion-pickup, sputtering and thermal escape.

Table 5 also shows $\mathrm{Fe}$ and $\mathrm{FeO}$. As can be seen for both - the molecule and atomic iron $-\lambda$ is clearly above $\lambda_{\text {crit }}$ for all magma ocean surface temperatures, meaning that Fe only escapes through Jeans escape and potentially through nonthermal escape processes, while $\mathrm{Mg}$ and $\mathrm{Si}$ can escape more easily. Therefore it seems likely that for planetary embryos that are not capable of keeping a stable steam atmosphere $\mathrm{Fe}$ might be enriched against $\mathrm{Mg}$ and $\mathrm{Si}$, even if the body is not differentiated.

Recently Young et al. (2019) investigated the escape of a rock-vapour atmosphere with an average molecular mass of $34 \mathrm{~g} / \mathrm{mol}$ that was outgassed from a planetary embryo with a radius of $700 \mathrm{~km}$ and 0.5 Pluto-masses (i.e. with $\left.0.088 \mathrm{M}_{\mathrm{Moon}}\right)$, and a magma ocean surface temperature of $1830 \mathrm{~K}$. These authors assumed that this outgassed atmosphere obtains a hydrostatic equilibrium and builds up a stable atmosphere as an equilibrium between outgassing 

a radiative layer (stratosphere), and a collision-less exosphere with a relative depth controlled by the mass and surface temperature. However, under these planetary parameters the assumption of Young et al. (2019) of a hydrostatic equilibrium is physically not valid.

610 as the gravity of the body, are balanced by the pressure-gradient force of the atmosphere. By using the parameters given in Young et al. (2019), the escape velocity on the surface of such an embryo approximately equals the mean speed of the Maxwell-Boltzmann distribution of the bulk rock-vapor atmosphere. This corresponds to a Jeans escape parameter on the surface of $\lambda=1.38$. As already described above, such an atmosphere is by no means in a hydrostatic equilibrium but clearly in a regime of strong hydrodynamic escape (e.g. Chamberlain, 1963; Bauer \& Lammer, 2004; Volkov et al., 2011; Erkaev et al., 2014). Furthermore, the pressure in their assumed convective layer that is in the order of ${ }_{620} \leq 2 \times 10^{-8}$ bar is less than a typical atmospheric pressure at the homopause level, meaning that eddy diffusion is not fulfilled and the atmosphere cannot be assumed to be well mixed; each species follows its own scale height. Under such conditions gravity cannot prevent the atmosphere from diffusing into space and no stable atmosphere can be maintained and the outgassing flux approximately equals the escape rate.

Furthermore, we tried to simulate a steam atmosphere with our 1D radiative-convective atmosphere model (see Section 3) with the same parameters as in the study of Young et al. (2019) for a protoplanet with a radius of $700 \mathrm{~km}$, a mass of $0.5 \mathrm{M}_{\text {Pluto }}$, and a surface temperature of $1830 \mathrm{~K}$ and found a divergence in hydrostatic altitude above $P / P_{\text {surf }}=0.2$ (see Fig. 9 , meaning that atmospheric build-up is impossible in such a case due to a direct escape of the molecules at thermal or sound speed unless there is a steady supply from the surface/interior to compensate for these losses. For a silicate atmosphere around such bodies, this means that the entire moderately volatile elements in 635 the magmatic layer will eventually evaporate. 
The assumption of a hydrostatic equilibrium in the study of Young et al. (2019) is thus only justified for cases in which the Jeans escape parameters of the outgassed elements at the surface of an embryo exceed $\lambda \approx 6$ (Erkaev et al., 2015; Volkov et al., 2011).

We additionally applied an adopted version of the 1D hydrodynamic atmosphere model as described in Erkaev et al. (2015) and Erkaev et al. (2016) to a similar silicate atmosphere as assumed by Young et al. (2019) with an average atomic mass of $34 \mathrm{amu}$. Here, Fig. 10a shows the evolution of the atmospheric temperature (red and blue lines) and the corresponding $\lambda$ (green and black) as retrieved by our model and by Young et al. (2019), respectively. In our case, the temperature is decreasing much more rapidly than in the model of Young et al. (2019) due to the adiabatic cooling of the hydrdynamically outflow of the bulk gas. On the top of the atmosphere at above ${ }_{650} \approx \mathbf{9} \mathbf{R}_{\text {body }}$ the EUV flux, which we scaled to the value expected for the early solar system after the solar nebula dissipated (Tu et al., 2015), is absorbed, heats the gas and builds up a thermosphere layer. The escaping hydrodynamic flow is supersonic just from the lower boundary for a low $\lambda$ of $\approx 1.4$. From the simulation, we also see that the density is decreasing rather slow, still being large at the upper boundary. Because of that, the EUV flux cannot penetrate deep into the atmosphere and, thus, it is absorbed in the upper atmospheric layers. Here, the absorbed EUV flux produces heating and a growth in temperature and pressure. The interaction between the supersonic hydrodynamic flow and the heated region leads to an appearance of a standing shock. At the shock front one can see a velocity drop (see Fig. 10a) due to a density rise, but the loss rate remains constant. In case of very a low lambda $(\approx 1)$, the interaction between the escaping supersonic flow from the atmosphere and incoming EUV flux results in the appearance of a stationary standing shock wave at the top of the atmosphere. 
However, concerning the equilibrium of outgassing and escape rates that converges into a particular surface pressure, the results of Young et al. (2019), even though the assumption of a hydrostatic atmosphere is physically not valid under such conditions, do not differ much from ours as shown in Fig. 11. While Young et al. (2019) obtained a pressure of $\approx 1 \times 10^{-8}$ bar and an evaporation and escape rate of $\approx 7.5 \times 10^{32} \mathbf{s}^{-1}$, we retrieve values of $\approx 2 \times 10^{-8}$ bar for the pressure and $\approx 5.5 \times 10^{32} \mathrm{~s}^{-1}$ for the evaporation and escape rate. The reason for the only minor divergence can be found in the fact that such a hot and low-mass body just cannot hold the gas and everything evaporates into space, leading to similar results.

\section{Discussion and conclusion}

The different escape scenarios are summarized in Fig. 12. While for small planetary embryos with masses of $M \leq 1 M_{\text {Moon }}$ no dense atmosphere can build up due to the strong escape of volatile elements that are outgassed from the magma ocean, more massive embryos will build up a steam atmosphere during magma ocean solidification which will later-on escape due to strong EUV driven hydrodynamic escape. The escaping $\mathrm{H}$ atoms will drag away heavier elements which reach the upper atmosphere in dependence of their weight.

In Fig. 13 one can see the relation between the orbital location $d$ of the planetary embryo, the magma ocean surface temperature and the amount of loss of $\mathrm{K}$. The reason for the decrease in escape for larger orbit distances is the declining intensity of the EUV flux which decreases with $1 / d^{2}$. For all simulated cases the entire budget of outgassed moderately volatile elements and noble gases from the magma ocean are lost due to hydrodynamic escape as long as the embryo is located within about $d=2 \mathrm{AU}$, which is the reason why the profiles of Fig. 13 flatten for low heliocentric distances. For a complete loss, however, the timescale of the condensation matters which

is related to the distance and the lifetime of a possible shallow magma ocean 
below the steam atmosphere. If the surface cools down, the remaining $\mathrm{H}_{2} \mathrm{O}$ vapor condenses, escape decreases and part of the volatile elements will remain at the embryo.

It has further to be noted that even though these planetary embryos can lose most of their noble gases, the initial fractionation of ${ }^{20} \mathrm{Ne} /{ }^{22} \mathrm{Ne}$ and ${ }^{36} \mathrm{Ar} /{ }^{38} \mathrm{Ar}$ won't change significantly.

Table 7: Escape duration for cases with an atmospheric pressure of 50 bar $\mathrm{H}_{2} \mathrm{O}$ and $5 \mathrm{bar}$ $\mathrm{CO}_{2}$ and a $T_{\text {surf }}$ of $1500 \mathrm{~K}$ for planetary embryos with masses of $0.5 M_{\text {Mars }}$ (cases I-III from Table 2), 1.0 $M_{\text {Mars }}$ (cases V-VII from Table 3), and 0.5 $M_{\text {Mars }}$ (cases IX-XI from Table 4) and for orbital locations $d$ between 0.5 and $1 \mathrm{AU}$.

\begin{tabular}{ccc} 
cases & $\begin{array}{c}\text { slow rotator } \\
\text { time } \\
{[\mathrm{Myr}]}\end{array}$ & $\begin{array}{c}\text { moderate rotator } \\
\text { time } \\
{[\mathrm{Myr}]}\end{array}$ \\
\hline \hline I & 0.26 & 0.15 \\
II & 0.54 & 0.29 \\
III & 1.11 & 0.61 \\
\hline V & 0.77 & 0.42 \\
VI & 1.51 & 0.83 \\
VII & 3.09 & 1.69 \\
\hline IX & 1.15 & 0.63 \\
X & 2.27 & 1.24 \\
XI & 4.66 & 2.55 \\
\hline
\end{tabular}

The escape of rock-forming elements which has been obtained with these simulations might ultimately also alter the respective $\mathrm{Fe} / \mathrm{Mg}$ and $\mathrm{Si} / \mathrm{Fe}$ ratios not only of these proto-planetary embryos but also of the planets that will accrete these embryos. Carter et al. (2015) and Bonsor et al. (2015) tried to reproduce the present-day $\mathrm{Mg} / \mathrm{Fe}$ and $\mathrm{Fe} / \mathrm{Si}$ ratios via collisional erosion of differentiated bodies with the assumption of the Earth being accreted from chondritic material. In their simulations, however, Earth can only be reproduced with significant dynamical excitation of the growing embryos (Carter et al., 
$10 \%$ silicon by mass (Bonsor et al., 2015). Since our mechanism of thermal escape might also partially account for the very high $\mathrm{Fe} / \mathrm{Mg}$ and low $\mathrm{Si} / \mathrm{Fe}$ ratios of the present-day Earth, such extreme dynamical scenarios or core $\mathrm{Si}$ abundances might therefore not be necessary. In this context, however, further needed in the future. Even though $\mathrm{Fe}$ is heavier than $\mathrm{Mg}$ and can, thus, less easily escape (see also Table 5), it is on the other hand more volatile than $\mathrm{Mg}$ and hence most likely more abundant in such an escaping atmosphere.

The significant loss of the radioactive heat producing isotope ${ }^{40} \mathrm{~K}$ will take an important role in the development of tectonics and hence the potential habitability of terrestrial planets. Extrasolar Systems with different stellar EUV evolution and planetary accretion history than the Solar System might ultimately lead to significantly different compositional abundances of heat producing elements in their respective planets which can affect their geophysical properties such as their tectonic regime as well as their potential magnetic dynamo (e.g. ??). Whether such planets will end up with more or less ${ }^{40} \mathrm{~K}$ as e.g. Earth might therefore be a crucial key factor in them being habitable or not.

Since the reservoir of elements that potentially can outgas and escape depend on the magma ocean depth, the depletion of moderately volatile elements might change as well, if the depth of the magma ocean is different than assumed in our study. It should, however, also be noted that the ratios and depletions of the volatile rock-forming elements calculated in this study most likely represent an upper value for the given magma ocean depth. The two main reasons lost to space, it is unclear whether $100 \%$ of the respective species will indeed be incorporated into the steam atmosphere or if a fraction remains inside the magma ocean. ii) The outgassed elements have to overcome a bottle neck from the lower atmosphere to the thermosphere, i.e. the so-called cold-trap, where 
early phase of the Solar System, however, might cause shock waves and turbulently mix the atmosphere which can lift these elements above the cold-trap into the thermosphere (e.g. Genda \& Abe, 2003; Trigo-Rodríguez \& Martín-Torres, 2013). Alternatively, one should not forget that bigger planetary embryos form from the collisions of smaller ones. As discussed in Section 4.2, these building blocks also underwent strong depletion during their boil-off phases so that one can expect the final composition to be a mixture of these complex interactions which is planned to be studied in more detail in the future. For noble gases, however, the cold-trap in steam atmospheres of larger bodies is not existing due to their low condensation temperatures.

From the results shown in Section 4 one can see that for the loss of elements the difference between a slow and a moderate rotator consists only in the duration of the loss (Table 7). It doesn't matter whether the Sun was a slow, moderate or fast rotator, planetary embryos of up to $1.5 \mathrm{M}_{\text {Mars }}$ that are orbiting in close distance to the Sun can either lose all volatiles that are outgassed from the magma ocean (within $\approx 2 \mathrm{AU}$ ) or at least a significant amount of the outgassed material for orbital location to several AU. A growing protoplanet might therefore accrete significantly volatile depleted material after the stellar nebula dissipated and during the so-called giant impact phase. Our results indicate in agreement with Sossi et al. (2019) and Lammer et al. (2019) that Earth's volatile inventory is a product of the accretion of various smaller planetary embryos that had experienced different levels of volatile escape. As Lammer et al. (2019) has shown in agreement with Marty (2012) through analysis of the present-day Ne and Ar isotopic ratios, Earth accreted $\approx 0.95 M_{\text {Earth }}$ from building blocks that were extremely depleted in volatiles which illustrates that most of the accreted material has indeed suffered from such extreme escape processes. A late accretion of chondritic material via large impactors might therefore not necessarily lead to a volatile rich planet.

Acknowledgements M.R. Benedikt and H. Lammer acknowledge support from the Austrian Forschungsförderungsgesellschaft FFG project RASEN. M. Leitzinger and P. Odert acknowledge support of the FWF projects P27256-N27 
and P30949-N36. N.V.E. acknowledges the Russian Science Foundation grant No 18-12-00080. H. Lammer and M. Scherf thank B. Fegley and K. Lodders for discussions on magma ocean-related outgassing of rock-forming elements.

775 Finally, we thank Paolo Sossi and an anonymous referee for their constructive comments which helped to improve the manuscript significantly. 


\section{References}

Agee, C. B., \& Draper, D. S. (2004). Experimental constraints on the origin of Martian meteorites and the composition of the Martian mantle. Earth and Planetary Science Letters, 224, 415-429. doi:10.1016/j .eps1.2004.05.022.

Albarède, F., \& Blichert-Toft, J. (2007). The split fate of the early Earth, Mars, Venus, and Moon. Comptes Rendus Geoscience, 339, 917-927. doi:10.1016/ j.crte.2007.09.006.

Bauer, S. J., \& Lammer, H. (2004). Planetary aeronomy : atmosphere environments in planetary systems.

Birnstiel, T., Fang, M., \& Johansen, A. (2016). Dust evolution and the formation of planetesimals. Space Science Reviews, 205, 41-75. URL: https://doi. org/10.1007/s11214-016-0256-1. doi:10.1007/s11214-016-0256-1.

Blum, J. (2018). Dust evolution in protoplanetary discs and the formation of planetesimals. Space Science Reviews, 214, 52. URL: https://doi.org/10. 1007/s11214-018-0486-5. doi:10.1007/s11214-018-0486-5.

Bollard, J., Connelly, J. N., Whitehouse, M. J., Pringle, E. A., Bonal, L., Jørgensen, J. K., Nordlund, Å., Moynier, F., \& Bizzarro, M. (2017). Early formation of planetary building blocks inferred from $\mathrm{Pb}$ isotopic ages of chondrules. Science Advances, 3, e1700407. doi:10.1126/sciadv.1700407. arXiv: 1708.02631

Bonsor, A., Leinhardt, Z. M., Carter, P. J., Elliott, T., Walter, M. J., \& Stewart, S. T. (2015). A collisional origin to Earth's non-chondritic composition? Icarus, 247, 291-300. doi:10.1016/j.icarus.2014.10.019. arXiv: 1410.3421 .

Boujibar, A., Andrault, D., Bolfan-Casanova, N., Bouhifd, M. A., \& Monteux, J. (2015). Cosmochemical fractionation by collisional erosion during the Earth's accretion. Nature Communications, 6, 8295. doi:10.1038/ncomms9295. 
Bouvier, L. C., Costa, M. M., Connelly, J. N., Jensen, N. K., Wielandt, D., Storey, M., Nemchin, A. A., Whitehouse, M. J., Snape, J. J. J. F., Bellucci, Moynier, F., Agranier, A., Gueguen, B., Schönbächler, M., \& Bizzarro, M. (2018). Evidence for extremely rapid magma ocean crystallization and crust formation on Mars. Nature, 558, 568-589. doi:10.1038/s41586-018-0222-z.

Brasser, R. (2013). The Formation of Mars: Building Blocks and Accretion Time Scale. Space Sci. Rev., 174, 11-25. doi:10.1007/s11214-012-9904-2. Carter, P. J., Leinhardt, Z. M., Elliott, T., Walter, M. J., \& Stewart, S. T. (2015). Compositional Evolution during Rocky Protoplanet Accretion. ApJ, 813, 72. doi:10.1088/0004-637X/813/1/72. arXiv:1509. 07504.

Chamberlain, J. W. (1963). Planetary coronae and atmospheric evaporation. Planet. Space Sci., 11, 901-960. doi:10.1016/0032-0633(63)90122-3.

Chassefière, E. (1996a). Hydrodynamic escape of hydrogen from a hot waterrich atmosphere: The case of Venus. J. Geophys. Res., 101, 26039-26056. doi:10.1029/96JE01951.

Chassefière, E. (1996b). Hydrodynamic Escape of Oxygen from Primitive Atmo820 spheres: Applications to the Cases of Venus and Mars. Icarus, 124, 537-552. doi:10.1006/icar.1996.0229.

Christian, D. J., \& Athioudakis, M. (2002). High-Resolution Optical Observations of Extreme-Ultraviolet-selected Active Late-type Stars. Astrophys. J., 123, 2796-2805. doi:10.1086/339702.

Dauphas, N., \& Pourmand, A. (2011). Hf-W-Th evidence for rapid growth of Mars and its status as a planetary embryo. Nature, 473, 489-492. doi:10. 1038/nature10077.

Dong, C., Lee, Y., Ma, Y., Lingam, M., Bougher, S., Luhmann, J., Curry, S., Toth, G., Nagy, A., Tenishev, V., Fang, X., Mitchell, D., Brain, D., \& 
Jakosky, B. (2018). Modeling Martian Atmospheric Losses over Time: Implications for Exoplanetary Climate Evolution and Habitability. ApJ, 859, L14. doi:10.3847/2041-8213/aac489. arXiv:1805.05016.

Elkins-Tanton, L. T. (2008). Linked magma ocean solidification and atmospheric growth for Earth and Mars. Earth Planet. Sci. Lett., 271, 181-191. doi:10. 1016/j.epsl.2008.03.062.

Elkins-Tanton, L. T. (2008). Linked magma ocean solidification and atmospheric growth for Earth and Mars. Earth and Planetary Science Letters, 271, 181191. doi:10.1016/j.epsl.2008.03.062.

Elkins-Tanton, L. T. (2012). Magma Oceans in the Inner Solar System. Annu. Rev. Earth Planet. Sci., 40, 113-139. URL: http:// Www . annualreviews .org/doi/10.1146/annurev-earth-042711-105503. doi:10.1146/annurev-earth-042711-105503.

Elkins-Tanton, L. T., Weiss, B. P., \& Zuber, M. T. (2011). Chondrites as samples of differentiated planetesimals. Earth Planet. Sci. Lett., 305, 1-10. URL: http://dx.doi.org/10.1016/j.epsl.2011.03.010. doi:10.1016/j . epsl.2011.03.010.

Erkaev, N. V., Lammer, H., Elkins-Tanton, L. T., Stökl, A., Odert, P., Marcq, E., Dorfi, E. A., Kislyakova, K. G., Kulikov, Y. N., Leitzinger, M., \& Güdel, M. (2014). Escape of the martian protoatmosphere and initial water inventory. Planet. Space Sci., 98, 106-119. doi:10.1016/j.pss.2013.09.008. arXiv:1308.0190.

Erkaev, N. V., Lammer, H., Odert, P., Kislyakova, K. G., Johnstone, C. P., Güdel, M., \& Khodachenko, M. L. (2016). EUV-driven mass-loss of protoplanetary cores with hydrogen-dominated atmospheres: the influences of ionization and orbital distance. MNRAS, 460, 1300-1309. doi:10.1093/mnras/ stw935. arXiv:1601.00452. 
Erkaev, N. V., Lammer, H., Odert, P., Kulikov, Y. N., \& Kislyakova, K. G. (2015). Extreme hydrodynamic atmospheric loss near the critical thermal escape regime. MNRAS, 448, 1916-1921. doi:10.1093/mnras/stv130. arXiv: 1506.06592

Erkaev, N. V., Lammer, H., Odert, P., Kulikov, Y. N., Kislyakova, K. G., Khodachenko, M. L., Güdel, M., Hanslmeier, A., \& Biernat, H. (2013). XUVExposed, Non-Hydrostatic Hydrogen-Rich Upper Atmospheres of Terrestrial Planets. Part I: Atmospheric Expansion and Thermal Escape. Astrobiology, 13, 1011-1029. doi:10.1089/ast.2012.0957. arXiv:1212 . 4982.

Eymet, V., Fournier, R., Dufresne, J. L., Lebonnois, S., Hourdin, F., \& Bullock, M. A. (2009). Net exchange parameterization of thermal infrared radiative transfer in Venus' atmosphere. J. Geophys. Res. E Planets, 114. doi:10. 1029/2008JE003276.

Fegley, B., Jr., Jacobson, N. S., Williams, K. B., Plane, J. M. C., Schaefer, L., \& Lodders, K. (2016). Solubility of Rock in Steam Atmospheres of Planets. ApJ, 824, 103. doi:10.3847/0004-637X/824/2/103. arXiv:1602.00658.

Fish, R. A., Goles, G. G., \& Anders, E. (1960). The Record in the Meteorites. III. on the Development of Meteorites in Asteroidal Bodies. ApJ, 132, 243. doi:10.1086/146918.

Fossati, L., Erkaev, N. V., Lammer, H., Cubillos, P. E., Odert, P., Juvan, I., Kislyakova, K. G., Lendl, M., Kubyshkina, D., \& Bauer, S. J. (2017). Aeronomical constraints to the minimum mass and maximum radius of hot low-mass planets. A\&A, 598, A90. doi:10.1051/0004-6361/201629716. arXiv: 1612.05624.

Genda, H., \& Abe, Y. (2003). Survival of a proto-atmosphere through the stage of giant impacts: the mechanical aspects. Icarus, 164, 149-162. doi:10.1016/ S0019-1035(03) 00101-5. 
Guo, J. H. (2019). The effect of photoionization on the loss of water of the planet. The Astrophysical Journal, 872. doi:10.3847/1538-4357/aaffd4.

Haar, L., Gallagher, J. S. I., \& Kell, G. S. (1984). Nbs/nrc steam tables: Thermodynamic and transport properties and computer programs for vapor and liquid states of water in si units,.

Hamano, K., Abe, Y., \& Genda, H. (2013). Emergence of two types of terrestrial planet on solidification of magma ocean. Nature, 497, 607-610. doi:10.1038/ nature12163.

Hin, R. C., Coath, C. D., Carter, P. J., Nimmo, F., Lai, Y.-J., Pogge von Strandmann, P. A. E., Willbold, M., Leinhardt, Z. M., Walter, M. J., \& Elliott, T. (2017). Magnesium isotope evidence that accretional vapour loss shapes planetary compositions. Nature, 549, 511-515. URL: http://www . nature.com/doifinder/10.1038/nature23899. doi:10.1038/nature23899.

Huebner, W. F., \& Mukherjee, J. (2015). Photoionization and photodissociation rates in solar and blackbody radiation fields. Planet. Space Sci., 106, 11-45. doi:10.1016/j.pss. 2014.11.022.

Hunten, D. M. (1975). Vertical Transport in Atmospheres. In B. M. McCormac (Ed.), Atmospheres of Earth and the Planets (p. 59). volume 51 of Astrophysics and Space Science Library. doi:10.1007/978-94-010-1799-2_4.

Hunten, D. M., Pepin, R. O., \& Walker, J. C. G. (1987). Mass fractionation in hydrodynamic escape. Icarus, 69, 532-549. doi:10.1016/0019-1035(87) 90022-4.

Kasting, J. F. (1988). Runaway and moist greenhouse atmospheres and the evolution of Earth and Venus. Icarus, 74, 472-494. doi:10.1016/0019-1035(88) 90116-9.

Kasting, J. F., \& Pollack, J. B. (1983). Loss of water from Venus. I. Hydrody910 namic escape of hydrogen. Icarus, 53, 479-508. doi:10.1016/0019-1035(83) 90212-9. 
Kazenas, E. K., Zviadadze, G. N., \& Bolshikh, M. A. (1985). Thermodynamics of sublimation, dissociation and gas-phase reactions of vapors over silicon dioxide. Metally, (pp. 46-48).

Kopparapu, R. K., Ramirez, R., Kasting, J. F., Eymet, V., Robinson, T. D., Mahadevan, S., Terrien, R. C., Domagal-Goldman, S., Meadows, V., \& Deshpande, R. (2013). Habitable zones around main-sequence stars: New estimates. Astrophys. J., 765. doi:10.1088/0004-637X/765/2/131. arXiv: 1301.6674 .

Koskinen, T. T., Harris, M. J., Yelle, R. V., \& Lavvas, P. (2013). The escape of heavy atoms from the ionosphere of HD209458b. I. A photochemicaldynamical model of the thermosphere. Icarus, 226, 1678-1694. doi:10.1016/ j.icarus. 2012.09.027. arXiv:1210.1536.

Krasnopolsky, V. A., \& Feldman, P. D. (2001). Detection of Molecular Hydrogen in theAtmosphere of Mars. Science, 294, 1914-1917. doi:10.1126/science. 1065569 .

Lammer, H., Erkaev, N. V., Fossati, L., Juvan, I., Odert, P., Cubillos, P. E., Guenther, E., Kislyakova, K. G., Johnstone, C. P., Lüftinger, T., \& Güdel, M. (2016). Identifying the 'true' radius of the hot sub-Neptune CoRoT-24b by mass-loss modelling. MNRAS, 461, L62-L66. doi:10.1093/mnrasl/slw095. arXiv: 1605.03595.

Lammer, H., Erkaev, N. V., Odert, P., Kislyakova, K. G., Leitzinger, M., \& Khodachenko, M. L. (2013). Probing the blow-off criteria of hydrogenrich 'super-Earths'. MNRAS, 430, 1247-1256. doi:10.1093/mnras/sts705. arXiv: 1210.0793.

Lammer, H., Leitzinger, M., Scherf, M., Odert, P., Burger, C., Kubyshkina, D., Johnstone, C., Maindl, T., Schfer, C., Gdel, M., Tosi, N., Nikolaou, A., Marcq, E., Erkaev, N., Noack, L., Kislyakova, K., Fossati, L., Pilat-Lohinger, E., Ragossnig, F., \& Dorfi, E. (2019). Constraining the early evolution of venus 
and earth through atmospheric ar, ne isotope and bulk $\mathrm{k} / \mathrm{u}$ ratios. Icarus, (p. 113551). URL: http://www.sciencedirect.com/science/article/ pii/S0019103519301290. doi:https://doi.org/10.1016/j.icarus. 2019. 113551.

Lebrun, T., Massol, H., Chassefière, E., Davaille, A., Marcq, E., Sarda, P., Leblanc, F., \& Brandeis, G. (2013). Thermal evolution of an early magma ocean in interaction with the atmosphere. J. Geophys. Res. E Planets, 118, 1155-1176. doi:10.1002/jgre. 20068.

Lichtenberg, T., Golabek, G. J., Dullemond, C. P., Schönbächler, M., Gerya, T. V., \& Meyer, M. R. (2018). Impact splash chondrule formation during planetesimal recycling. Icarus, 302, 27-43. doi:10.1016/j.icarus.2017. 11.004. arXiv: 1711.02103.

Lichtenberg, T., Golabek, G. J., Gerya, T. V., \& Meyer, M. R. (2016a). The effects of short-lived radionuclides and porosity on the early thermo-mechanical evolution of planetesimals. Icarus, 274, 350-365. doi:10.1016/j.icarus . 2016.03.004. arXiv:1603.05979.

Lichtenberg, T., Keller, T., Katz, R. F., Golabek, G. J., \& Gerya, T. V. (2019). Magma ascent in planetesimals: Control by grain size. Earth and Planetary Science Letters, 507, 154-165. doi:10.1016/j .epsl.2018.11.034. arXiv: 1802.02157.

Lichtenberg, T., Parker, R. J., \& Meyer, M. R. (2016b). Isotopic enrichment of forming planetary systems from supernova pollution. MNRAS, 462, 39793992. doi:10.1093/mnras/stw1929. arXiv:1608.01435.

Lodders, K. (2003). Solar System Abundances and Condensation Temperatures of the Elements. Astrophys. J., 591, 1220-1247. URL: http://stacks.iop. org/0004-637X/591/i=2/a=1220. doi:10.1086/375492. arXiv:1409.7398.

Lodders, K., Palme, H., \& Gail, H.-P. (2009). 4.4 Abundances of the elements in the Solar System, . VI, 712-770. URL: http://materials. 
springer.com/lb/docs/sm\{_\}lbs\{_\}978-3-540-88055-4\{_\}34.

doi:10.1007/978-3-540-88055-4_34. arXiv:0901.1149.

Maindl, T. I., Dvorak, R., Lammer, H., Güdel, M., Schäfer, C., Speith, R., Odert, P., Erkaev, N. V., Kislyakova, K. G., \& Pilat-Lohinger, E. (2015). Impact induced surface heating by planetesimals on early Mars. A\&A, 574, A22. doi:10.1051/0004-6361/201424256. arXiv:1405.5913.

Marcq, E. (2012). A simple 1-D radiative-convective atmospheric model de975 signed for integration into coupled models of magma ocean planets. J. Geophys. Res. E Planets, 117, 1-9. doi:10.1029/2011JE003912.

Marcq, E., Salvador, A., Massol, H., \& Davaille, A. (2017). Thermal radiation of magma ocean planets using a 1-D radiative-convective model of $\mathrm{H}_{2} \mathrm{O}-\mathrm{CO}_{2}$ atmospheres. Journal of Geophysical Research (Planets), 122, 1539-1553. doi:10.1002/2016JE005224.

Marty, B. (2012). The origins and concentrations of water, carbon, nitrogen and noble gases on Earth. URL: http://dx.doi .org/10.1016/j.epsl.2011.10. 040. doi:10.1016/j.epsl.2011.10.040. arXiv:1405.6336.

Massol, H., Hamano, K., Tian, F., Ikoma, M., Abe, Y., Chassefière, E., Davaille, A., Genda, H., Güdel, M., Hori, Y., Leblanc, F., Marcq, E., Sarda, P., Shematovich, V. I., Stökl, A., \& Lammer, H. (2016). Formation and Evolution of Protoatmospheres. Space Sci. Rev., 205, 153-211. doi:10.1007/s11214-016-0280-1.

Mlawer, E. J., Payne, V. H., Moncet, J. L., Delamere, J. S., Alvarado, M. J., 990 \& Tobin, D. C. (2012). Development and recent evaluation of the MT-CKD model of continuum absorption. Philos. Trans. R. Soc. A Math. Phys. Eng. Sci., 370, 2520-2556. doi:10.1098/rsta.2011.0295.

Morbidelli, A., \& Raymond, S. N. (2016). Challenges in planet formation. Journal of Geophysical Research (Planets), 121, 1962-1980. doi:10.1002/ 2016JE005088. arXiv: 1610.07202. 
Murthy, V. R., van Westrenen, W., \& Fei, Y. (2003). Experimental evidence that potassium is a substantial radioactive heat source in planetary cores. Nature, 423, 163-165. doi:10.1038/nature01560.

Nimmo, F., \& Kleine, T. (2015). Early Differentiation and Core Formation. Washington DC American Geophysical Union Geophysical Monograph Series, 212, 83-102. doi:10.1002/9781118860359.ch5.

Nimmo, F., Price, G. D., Brodholt, J., \& Gubbins, D. (2004). The influence of potassium on core and geodynamo evolution. Geophysical Journal International, 156, 363-376. doi:10.1111/j.1365-246X.2003.02157.x.

Odert, P., Lammer, H., Erkaev, N. V., Nikolaou, A., Lichtenegger, H. I., Johnstone, C. P., Kislyakova, K. G., Leitzinger, M., \& Tosi, N. (2018). Escape and fractionation of volatiles and noble gases from Mars-sized planetary embryos and growing protoplanets. Icarus, 30\%, 327-346. doi:10.1016/j.icarus . 2017.10.031. arXiv:1706.06988.

O'Neill, H. S. C., \& Palme, H. (2008). Collisional erosion and the non-chondritic composition of the terrestrial planets. Philosophical Transactions of the Royal Society of London Series A, 366, 4205-4238. doi:10.1098/rsta.2008.0111.

Öpik, E. J. (1963). Selective Escape of Gases? Geophysical Journal, 7, 490-506. doi:10.1111/j.1365-246X.1963.tb07091.x.

Owen, J. E., \& Wu, Y. (2016). Atmospheres of Low-mass Planets: The "Boil-off". ApJ, 817, 107. doi:10.3847/0004-637X/817/2/107. arXiv: 1506.02049

Parker, E. N. (1963). Interplanetary dynamical processes..

Pepin, R. O. (2006). Atmospheres on the terrestrial planets: Clues to origin 1020 and evolution. Earth and Planetary Science Letters, 252, 1-14. doi:10.1016/ j.epsl.2006.09.014. 
Philpotts, J. A., \& Schnetzler, C. C. (1970). Potassium, Rubidium, Strontium, Barium, and Rare-Earth Concentrations in Lunar Rocks and Separated Phases. Science, 167, 493-495. doi:10.1126/science.167.3918.493.

Pluriel, W., Marcq, E., \& Turbet, M. (2019). Modeling the albedo of Earthlike magma ocean planets with $\mathrm{H}_{2} \mathrm{O}-\mathrm{CO}_{2}$ atmospheres. Icarus, 317, 583-590. doi:10.1016/j.icarus.2018.08.023. arXiv:1809.02036.

Raymond, S. N., Kokubo, E., Morbidelli, A., Morishima, R., \& Walsh, K. J. (2014). Terrestrial Planet Formation at Home and Abroad. Protostars and Planets VI, (pp. 595-618). doi:10.2458/azu_uapress_ 9780816531240-ch026. arXiv:1312.1689.

Righter, K., \& Chabot, N. L. (2011). Moderately and slightly siderophile element constraints on the depth and extent of melting in early Mars. Meteoritics and Planetary Science, 46, 157-176. doi:10.1111/j.1945-5100.2010. $01140 \cdot \mathrm{x}$

Righter, K., Hervig, R. L., \& Kring, D. A. (1998). Accretion and core formation on Mars: molybdenum contents of melt inclusion glasses in three SNC meteorites. Geochim. Cosmochim. Acta, 62, 2167-2177. doi:10.1016/ S0016-7037 (98) 00132-X.

Safronov, V. S., \& Zvjagina, E. V. (1969). Relative Sizes of the Largest Bodies during the Accumulation of Planets. Icarus, 10, 109-115. doi:10.1016/ 0019-1035 (69) 90013-X.

Salvador, A., Massol, H., Davaille, A., Marcq, E., Sarda, P., \& Chassefière, E. (2017). The relative influence of $\mathrm{H} 2 \mathrm{O}$ and $\mathrm{CO} 2 \mathrm{on}$ the primitive surface conditions and evolution of rocky planets. J. Geophys. Res. Planets, 122, 1458-1486. doi:10.1002/2017 JE005286.

Schaefer, L., \& Elkins-Tanton, L. T. (2018). Magma oceans as a critical stage in the tectonic development of rocky planets. Philosophical Transactions of the 
Royal Society of London Series A, 376, 20180109. doi:10.1098/rsta.2018.

Sneep, M., \& Ubachs, W. (2005). Direct measurement of the Rayleigh scattering cross section in various gases. J. Quant. Spectrosc. Radiat. Transf., 92, 293310. doi:10.1016/j.jqsrt.2004.07.025.

Sossi, P. A., Klemme, S., O’Neill, H. S. C., Berndt, J., \& Moynier, F. (2019). 1075

Schaefer, L., \& Fegley, B. (2007). Outgassing of ordinary chondritic material and some of its implications for the chemistry of asteroids, planets, and satellites. Icarus, 186, 462-483. doi:10.1016/j.icarus.2006.09.002. arXiv: astro-ph/0606671.

Schaefer, L., \& Fegley, B. (2010). Chemistry of atmospheres formed during accretion of the Earth and other terrestrial planets. Icarus, 208, 438-448. URL: http://dx.doi.org/10.1016/j.icarus.2010.01.026. doi:10.1016/ j.icarus.2010.01.026. arXiv:0909.4050.

Schaefer, L., \& Fegley, B. (2017). Redox States of Initial Atmospheres Outgassed on Rocky Planets and Planetesimals. Astrophys. J., 843, 120. URL: http://stacks.iop.org/0004-637X/843/i=2/a=120?key= crossref.26a30fc9ab5e1d3e4988aa2e8b5fefdb. doi:10.3847/1538-4357/ aa784f.

Shematovich, V. I., Ionov, D. E., \& Lammer, H. (2014). Heating efficiency in hydrogen-dominated upper atmospheres. A\&A, 571, A94. doi:10.1051/ 0004-6361/201423573. arXiv:1409.0730.

Siebert, J., Sossi, P. A., Blanchard, I., Mahan, B., Badro, J., \& Moynier, F. (2018). Chondritic Mn/Na ratio and limited post-nebular volatile loss of the Earth. Earth and Planetary Science Letters, 485, 130-139. doi:10.1016/j. eps1.2017.12.042.

Evaporation of moderately volatile elements from silicate melts: experiments 
and theory. Geochim. Cosmochim. Acta, 260, 204-231. doi:10.1016/j.gca. 2019.06 .021$.

Stefani, S., Piccioni, G., Snels, M., Grassi, D., \& Adriani, A. (2013). Experimental CO2absorption coefficients at high pressure and high temperature. $J$. Quant. Spectrosc. Radiat. Transf., 117, 21-28. URL: http://dx.doi.org/ 10.1016/j.jqsrt.2012.11.019. doi:10.1016/j.jqsrt. 2012.11.019.

Tian, F., Kasting, J. F., Liu, H.-L., \& Roble, R. G. (2008a). Hydrodynamic planetary thermosphere model: 1. Response of the Earth's thermosphere to extreme solar EUV conditions and the significance of adiabatic cooling. Journal of Geophysical Research (Planets), 113, E05008. doi:10.1029/2007 JE002946.

Tian, F., Kasting, J. F., \& Solomon, S. C. (2009). Thermal escape of carbon from the early Martian atmosphere. Geophys. Res. Lett., 36, L02205. doi:10. 1029/2008GL036513.

Tian, F., Solomon, S. C., Qian, L., Lei, J., \& Roble, R. G. (2008b). Hydrodynamic planetary thermosphere model: 2. Coupling of an electron transport/energy deposition model. Journal of Geophysical Research (Planets), 113, E07005. doi:10.1029/2007 JE003043.

Tian, F., Toon, O. B., Pavlov, A. A., \& De Sterck, H. (2005). Transonic Hydrodynamic Escape of Hydrogen from Extrasolar Planetary Atmospheres. ApJ, 621, 1049-1060. doi:10.1086/427204.

Tonks, W. B., \& Melosh, H. J. (1993). Magma ocean formation due to giant impacts. J. Geophys. Res., 98, 5319-5333. doi:10.1029/92JE02726.

Trigo-Rodríguez, J. M., \& Martín-Torres, F. J. (2013). Implication of Impacts in the Young Earth Sun Paradox and the Evolution of Earth's Atmosphere. In J. M. Trigo-Rodriguez, F. Raulin, C. Muller, \& C. Nixon (Eds.), The Early Evolution of the Atmospheres of Terrestrial Planets (p. 85). volume 35 of Astrophysics and Space Science Proceedings. doi:10.1007/ 978-1-4614-5191-4_7. 
Tu, L., Johnstone, C. P., Güdel, M., \& Lammer, H. (2015). The extreme ultraviolet and X-ray Sun in Time: High-energy evolutionary tracks of a solar-like star. A\&A, 577, L3. doi:10.1051/0004-6361/201526146. arXiv: 1504.04546.

Turbet, M., Forget, F., Head, J. W., \& Wordsworth, R. (2017). 3D modelling of the climatic impact of outflow channel formation events on early Mars. Icarus, 288, 10-36. URL: http://dx.doi.org/10.1016/j.icarus.2017.01.024. doi:10.1016/j.icarus.2017.01.024. arXiv:1701.07886.

Turcotte, D. L., \& Schubert, G. (2002). Geodynamics.

Urey, H. C. (1955). The Cosmic Abundances of Potassium, Uranium, and Thorium and the Heat Balances of the Earth, the Moon, and Mars. Proceedings of the National Academy of Science, 41, 127-144. doi:10.1073/pnas.41.3.127.

Volkov, A. N., Johnson, R. E., Tucker, O. J., \& Erwin, J. T. (2011). Thermally Driven Atmospheric Escape: Transition from Hydrodynamic to Jeans Escape. ApJ, 729, L24. doi:10.1088/2041-8205/729/2/L24. arXiv:1009.5110.

Wakita, H., \& Schmitt, R. A. (1970). Lunar Anorthosites: Rare-Earth and Other Elemental Abundances. Science, 170, 969-974. doi:10.1126/science. 170.3961 .969 .

Wang, H., Weiss, B. P., Bai, X.-N., Downey, B. G., Wang, J., Wang, J., Suavet, C., Fu, R. R., \& Zucolotto, M. E. (2017). Lifetime of the solar nebula constrained by meteorite paleomagnetism. Science, 355, 623-627. doi:10.1126/science. aaf5043.

Watson, A. J., Donahue, T. M., \& Walker, J. C. G. (1981). The dynamics of a rapidly escaping atmosphere - Applications to the evolution of earth and Venus. Icarus, 48, 150-166. doi:10.1016/0019-1035(81)90101-9.

Wetherill, G. W. (1980). Formation of the terrestrial planets. ARA\&A, 18, 77-113. doi:10.1146/annurev . aa.18.090180.000453. 
Wood, J. a. (2005). The Chondrite Types and their Origins, . 341, 953-971.

Young, E. D., Shahar, A., Nimmo, F., Schlichting, H. E., Schauble, E. A., Tang, H., \& Labidi, J. (2019). Near-equilibrium isotope fractionation during planetesimal evaporation. Icarus, 323, 1-15. doi:10.1016/j.icarus.2019. 01.012.

Zahnle, K., Pollack, J. B., \& Kasting, J. F. (1990). Mass fractionation of noble gases in diffusion-limited hydrodynamic hydrogen escape. Icarus, 84, 502527. doi:10.1016/0019-1035(90)90050-J.

Zahnle, K. J., \& Kasting, J. F. (1986). Mass fractionation during transonic 1140 escape and implications for loss of water from Mars and Venus. Icarus, 68, 462-480. doi:10.1016/0019-1035(86) 90051-5. 


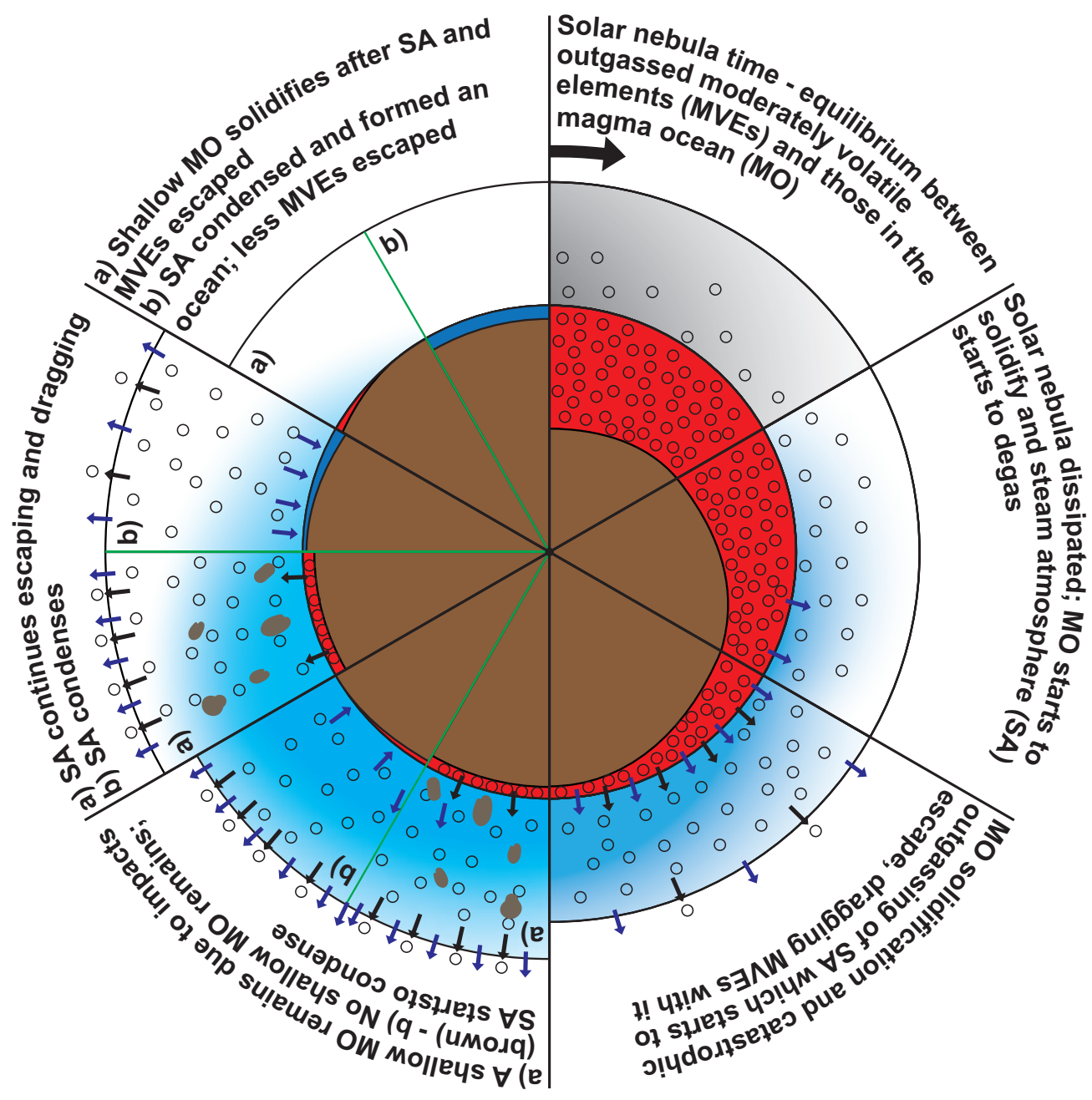

Figure 1: Illustration of the assumed evolutionary scheme from the nebula and magma ocean phase to escaping/condensing steam atmospheres. 

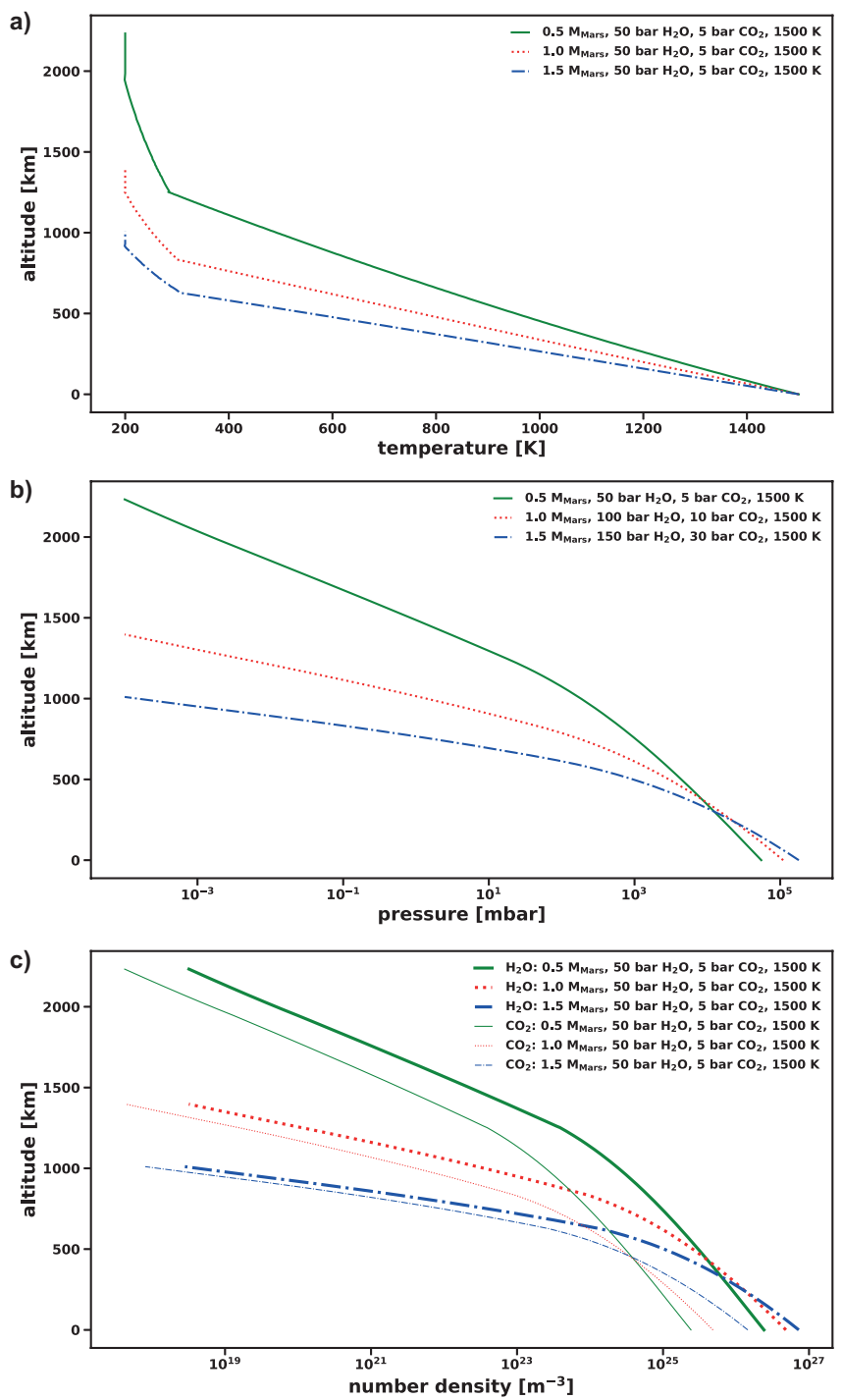

Figure 2: a) Temperature profiles for three selected steam atmospheres below the thermosphere as simulated with the 1D radiative-convective atmosphere model, i.e. for an orbital distance of $1.0 \mathrm{AU}, \mathrm{T}_{\mathrm{surf}}=1500 \mathrm{~K}$, and for a slow rotator, i.e. $M_{\mathrm{Emb}}=0.5 M_{\mathrm{Mars}}, 50 \mathrm{bar}$ $\mathrm{H}_{2} \mathrm{O}$ and 5 bar $\mathrm{CO}_{2}$ (Case III; upper panel), $M_{\text {Emb }}=1.0 M_{\text {Mars }}, 100$ bar $\mathrm{H}_{2} \mathrm{O}$ and $10 \mathrm{bar}$

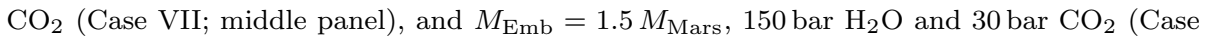
XI; lower panel). b) Pressure profiles for the same three cases. c) Number densities of $\mathrm{H}_{2} \mathrm{O}$ and $\mathrm{CO}_{2}$ for the same cases. 


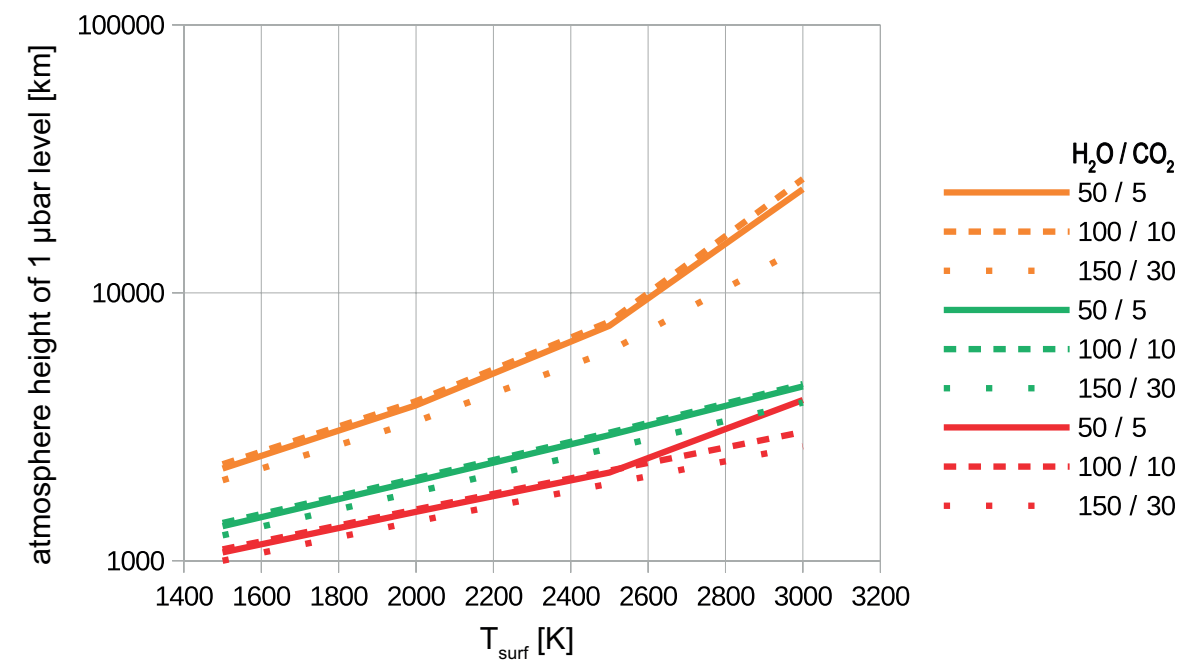

Figure 3: Relation between the surface temperature of the planetary embryo and the steam atmosphere height at the $1 \mu$ bar level for various atmospheric partial pressures $\left(\mathrm{H}_{2} \mathrm{O} / \mathrm{CO}_{2}\right)$. These heights depend mainly on $T_{\text {surf }}$ and not on the stellar luminosity, i.e. these are independent from the orbital location for these particular scenarios. Orange line: $0.5 M_{\text {Mars }}$; green line: $1.0 \mathrm{M}_{\mathrm{Mars}}$; red line: $1.5 \mathrm{M}_{\mathrm{Mars}}$. 


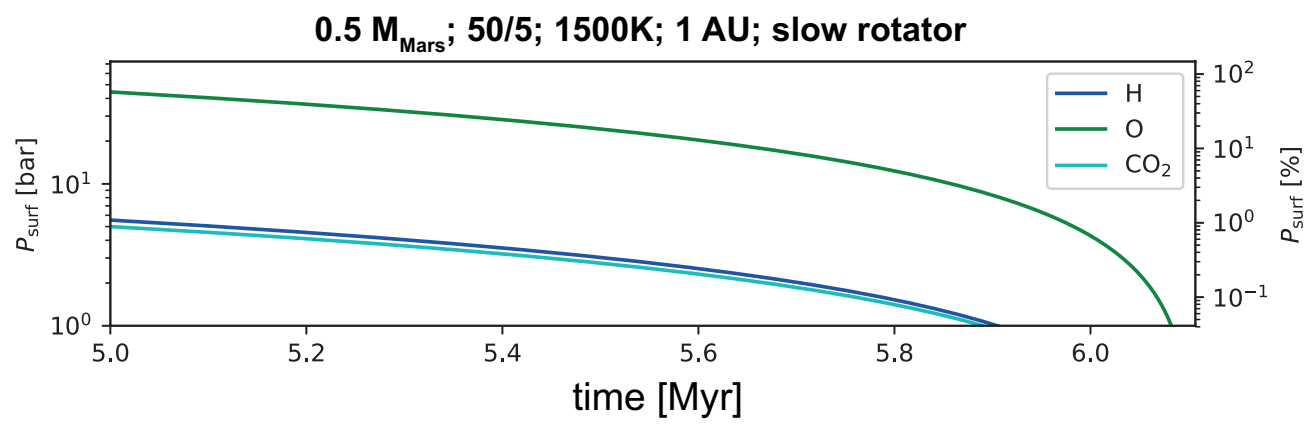

$1.0 \mathrm{M}_{\text {Mars }} ; 100 / 10 ; 1500 \mathrm{~K} ; 1 \mathrm{AU}$; slow rotator

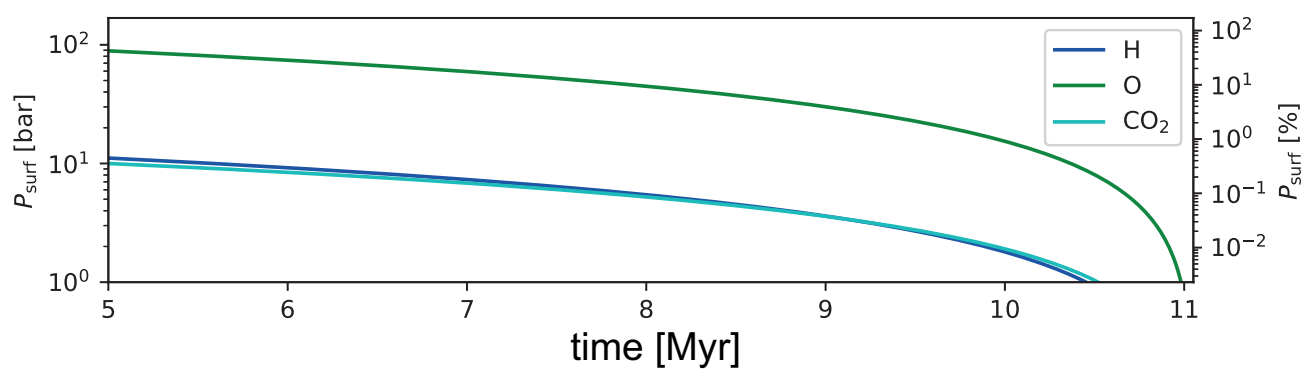

$1.5 \mathrm{M}_{\text {Mars }} ; 150 / 30 ; 1500 \mathrm{~K} ; 1 \mathrm{AU}$; slow rotator

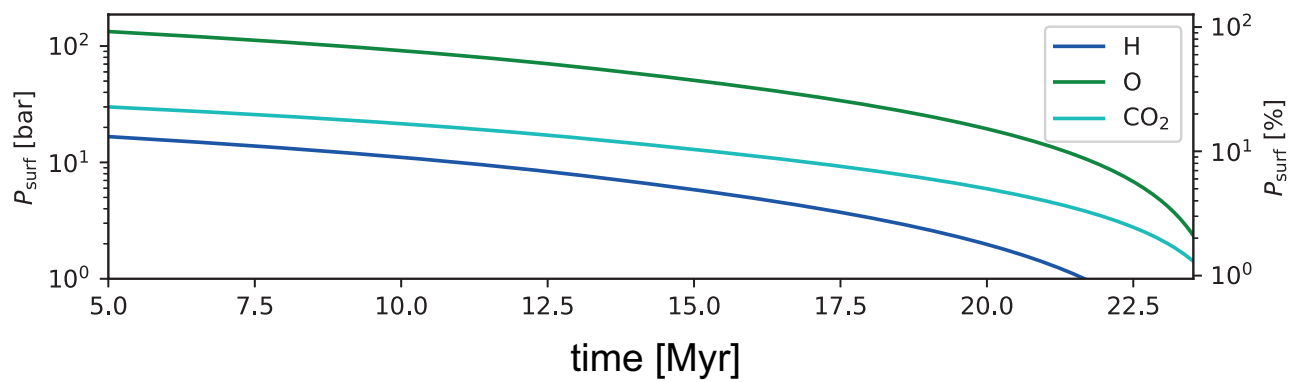

Figure 4: Atmospheric partial surface pressure evolution for different scenarios at an orbital distance of $1.0 \mathrm{AU}, \mathrm{T}_{\text {surf }}=1500 \mathrm{~K}$, and for a slow rotator, i.e $M_{\mathrm{Emb}}=0.5 M_{\text {Mars }}, 50 \mathrm{bar} \mathrm{H}_{2} \mathrm{O}$ and 5 bar $\mathrm{CO}_{2}$ (Case III; upper panel), $M_{\mathrm{Emb}}=1.0 M_{\mathrm{Mars}}, 100 \mathrm{bar} \mathrm{H}_{2} \mathrm{O}$ and $10 \mathrm{bar} \mathrm{CO}_{2}$ (Case VII; middle panel), and $M_{\mathrm{Emb}}=1.5 M_{\mathrm{Mars}}, 150$ bar $\mathrm{H}_{2} \mathrm{O}$ and $30 \mathrm{bar} \mathrm{CO}_{2}$ (Case XI; lower panel). 

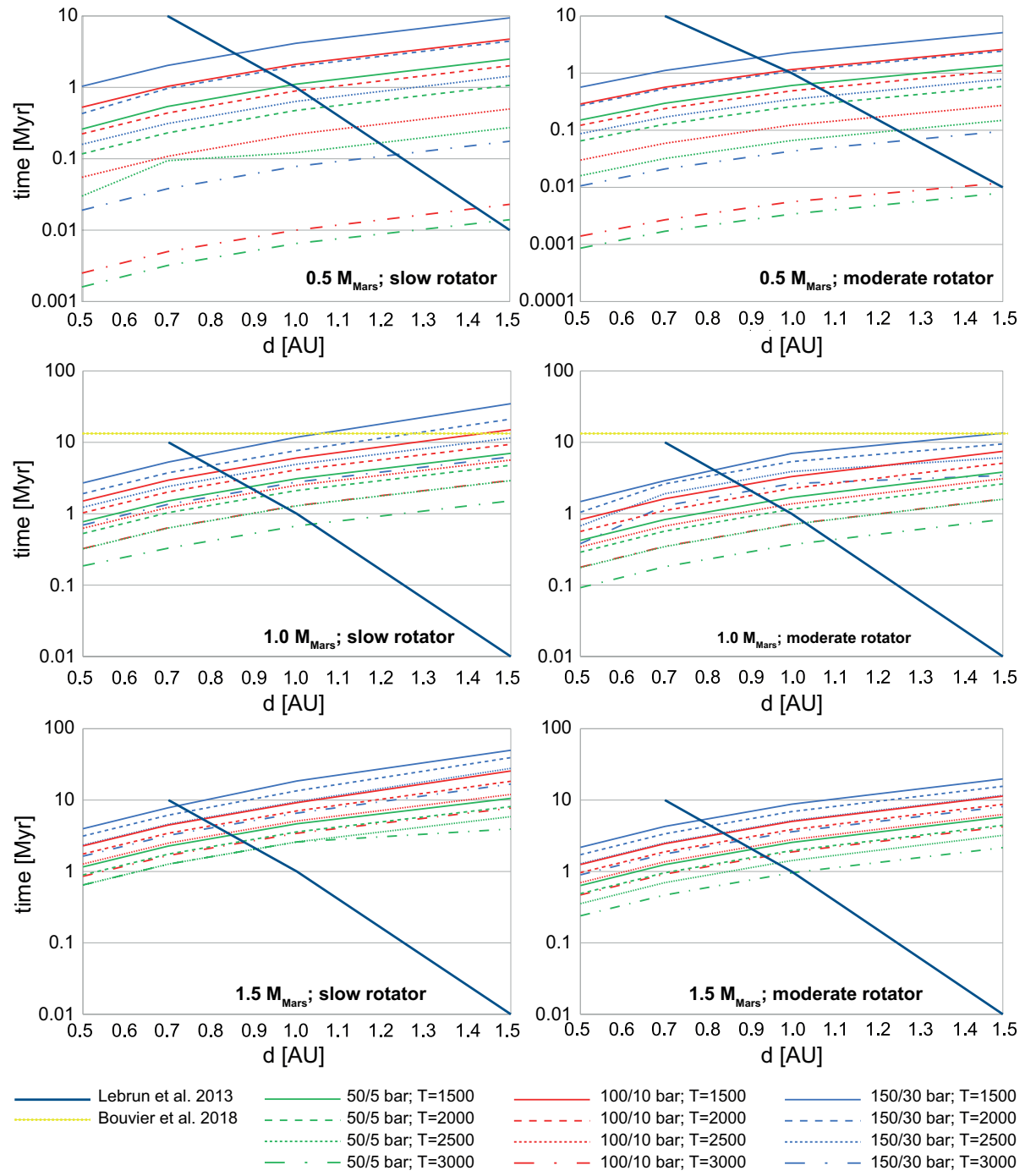

Figure 5: Duration for the total escape of the steam atmosphere for different protoplanetary masses, atmospheric pressures and temperatures for a slow and a moderate rotator in dependence of the orbital distance. The solid dark blue line in each panel shows the steam atmosphere solidification time based on the study of Lebrun et al. (2013); the dotted yellow

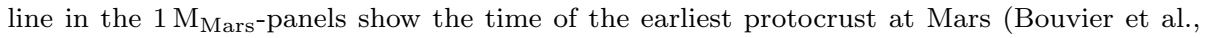
2018). 

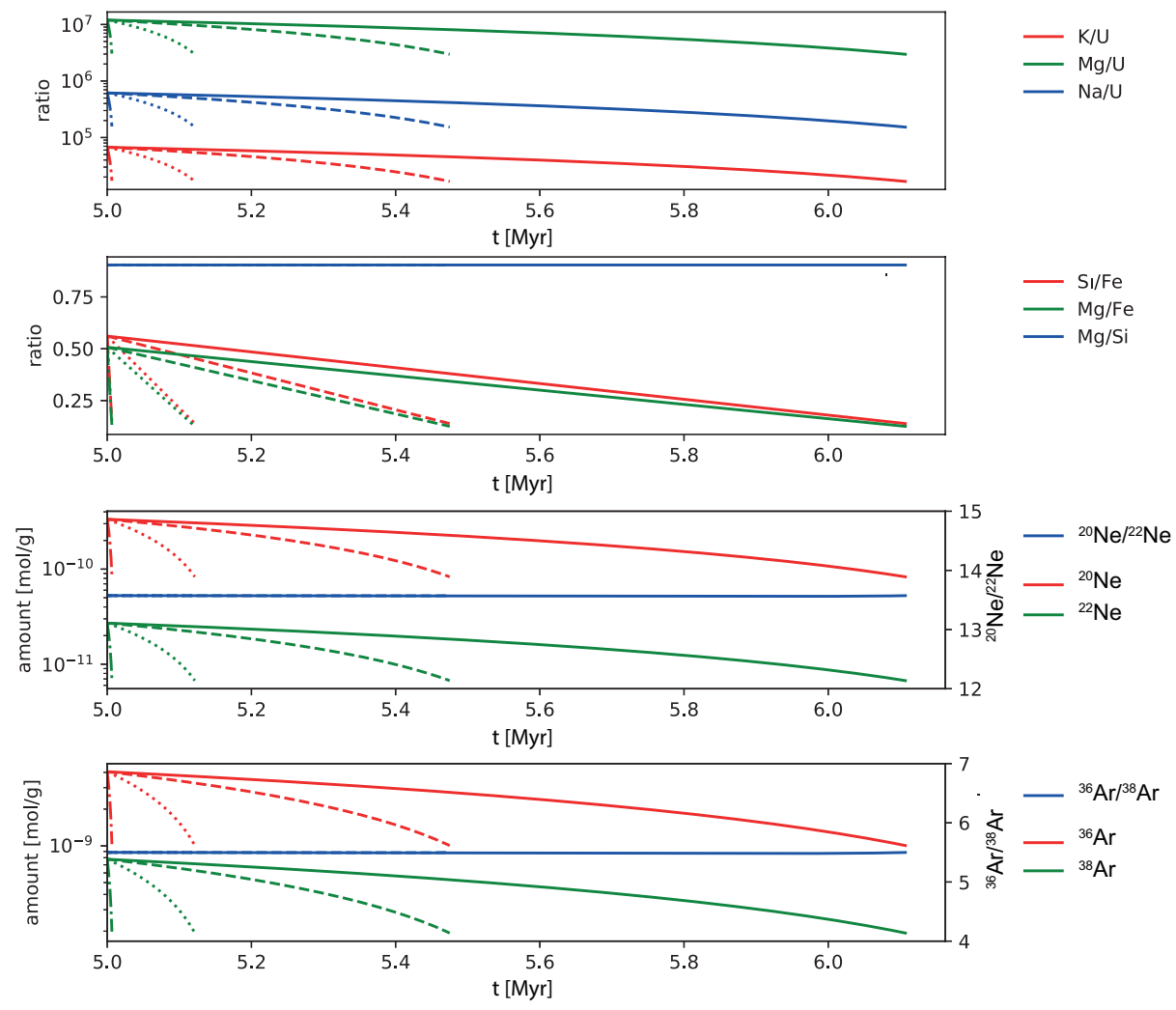

Figure 6: Results for $M_{\mathrm{Emb}}=0.5 M_{\mathrm{Mars}}$, an orbital distance of $1 \mathrm{AU}$ (case III), a slow rotator, 50 bar $\mathrm{H}_{2} \mathrm{O}$ and 5 bar $\mathrm{CO}_{2}$. About $75 \%$ of the total amount of the planetary embryo is lost, i.e. almost everything (>90\%) of the trace species that was contained in the magma ocean. Solid line: $T_{\text {surf }}=1500 \mathrm{~K}$; dashed line: $T_{\text {surf }}=2000 \mathrm{~K}$; dotted line: $T_{\text {surf }}=2500 \mathrm{~K}$; dash-dotted line: $T_{\text {surf }}=3000 \mathrm{~K}$. The different line-ends correspond to the loss-time of the steam atmosphere. The ratios are calculated for $\mathrm{mol} / \mathrm{g}$ and $\mathrm{U}$ as well as Fe were assumed to remain at the planet, i.e. their abundance was assumed to be constant. 

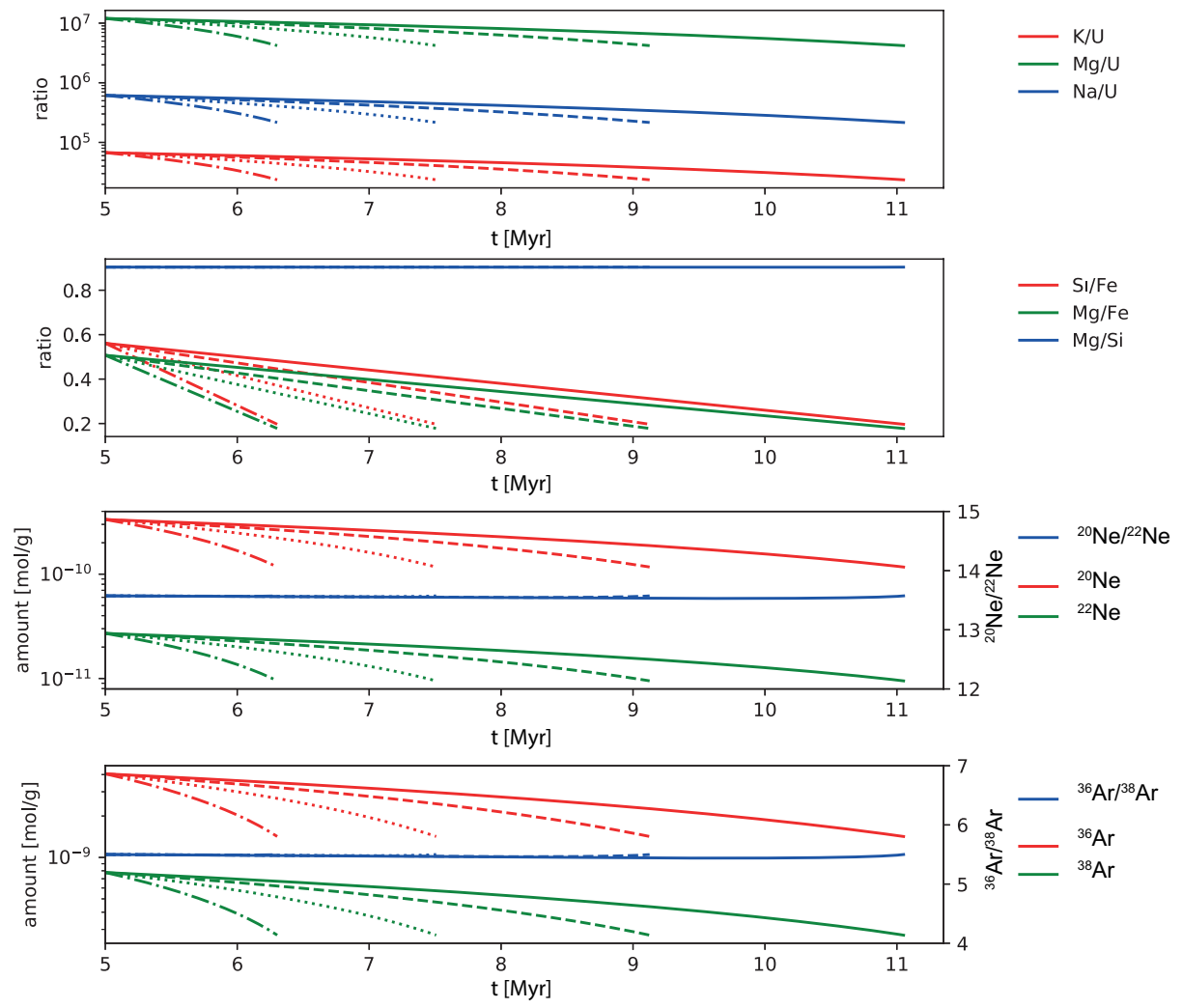

Figure 7: Results for $M_{\mathrm{Emb}}=1.0 M_{\mathrm{Mars}}$, an orbital distance of $1 \mathrm{AU}$ (case VII), a slow rotator, 100 bar $\mathrm{H}_{2} \mathrm{O}$ and 10 bar $\mathrm{CO}_{2}$. About $65 \%$ of the total amount of the planetary embryo is lost, i.e. almost everything $(>90 \%)$ of the trace species that was contained in the magma ocean. Solid line: $T_{\text {surf }}=1500 \mathrm{~K}$; dashed line: $T_{\text {surf }}=2000 \mathrm{~K}$; dotted line: $T_{\text {surf }}=2500 \mathrm{~K}$; dash-dotted line: $T_{\text {surf }}=3000 \mathrm{~K}$. The different line-ends correspond to the loss-time of the steam atmosphere. The ratios are calculated for $\mathrm{mol} / \mathrm{g}$ and $\mathrm{U}$ as well as Fe were assumed to remain at the planet, i.e. their abundance was assumed to be constant. 

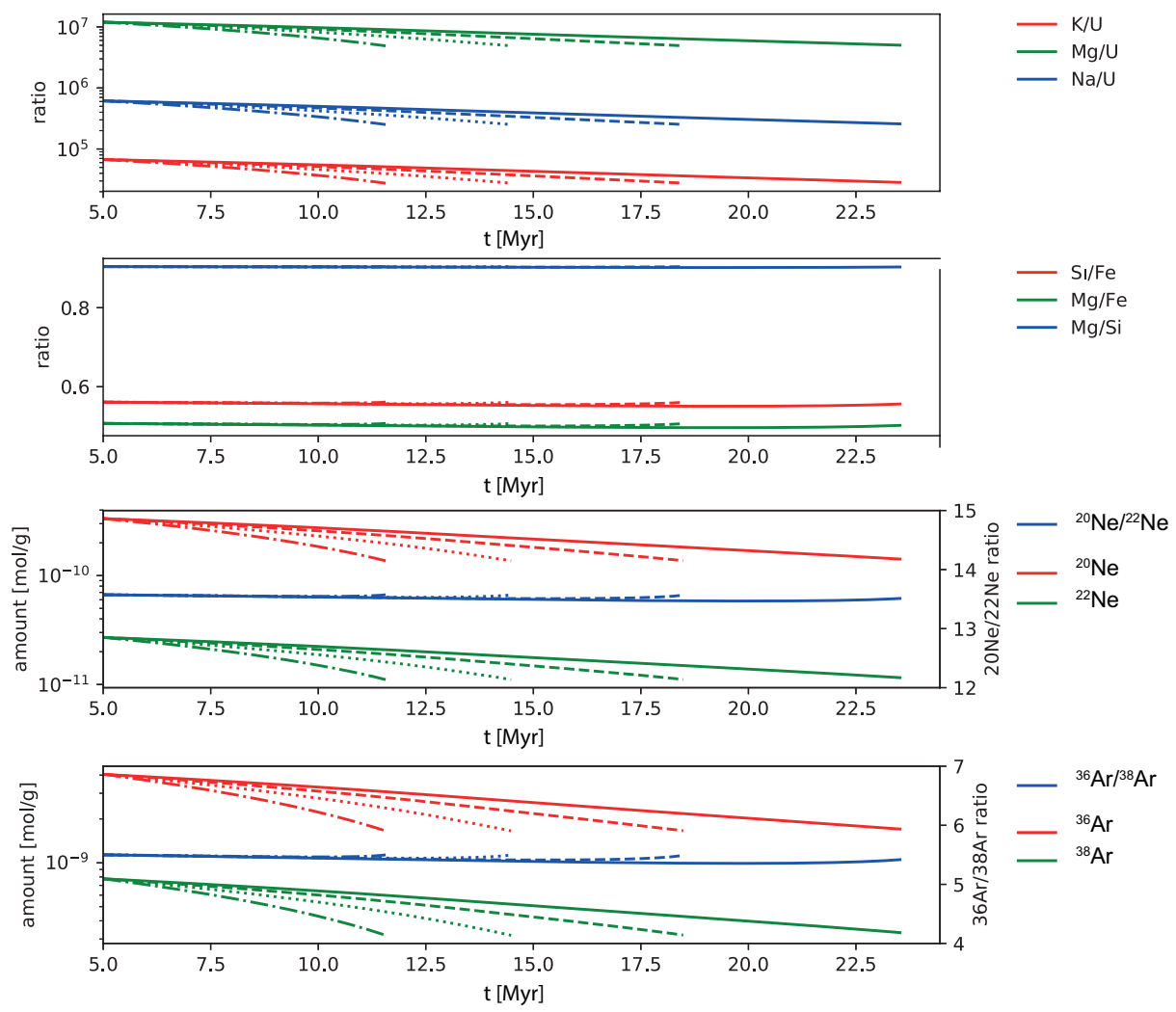

Figure 8: Results for $M_{\mathrm{Emb}}=1.5 M_{\mathrm{Mars}}$, an orbital distance of $1 \mathrm{AU}$ (case XI), a slow rotator, 150 bar $\mathrm{H}_{2} \mathrm{O}$ and 30 bar $\mathrm{CO}_{2}$. About $58 \%$ of the total amount of the planetary embryo is lost, i.e. almost everything $(>90 \%)$ of the trace species that was contained in the magma ocean. Solid line: $T_{\text {surf }}=1500 \mathrm{~K}$; dashed line: $T_{\text {surf }}=2000 \mathrm{~K}$; dotted line: $T_{\text {surf }}=2500 \mathrm{~K}$; dash-dotted line: $T_{\text {surf }}=3000 \mathrm{~K}$. The different line-ends correspond to the loss-time of the steam atmosphere. The ratios are calculated for $\mathrm{mol} / \mathrm{g}$ and $\mathrm{U}$ as well as Fe were assumed to remain at the planet, i.e. their abundance was assumed to be constant. 


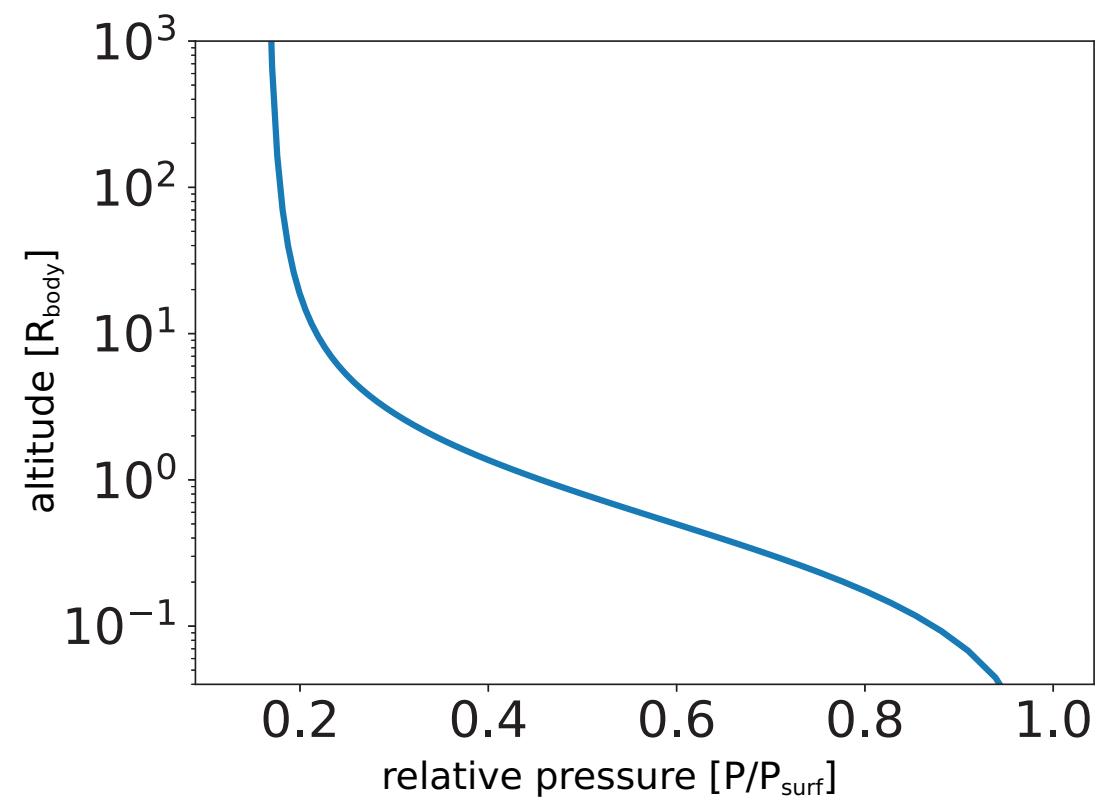

Figure 9: Simulation run with our 1D radiative-convective atmosphere model (as described in Section 3) of the case described in Young et al. (2019) with $M=0.5 \mathrm{M}_{\text {Pluto }}, R=700 \mathrm{~km}$ and $T_{\text {surf }}=700 \mathrm{~K}$. In our model the pressure does not drop to zero as altitude goes to infinity, i.e. no stable solution was found for this particular case. 
a)

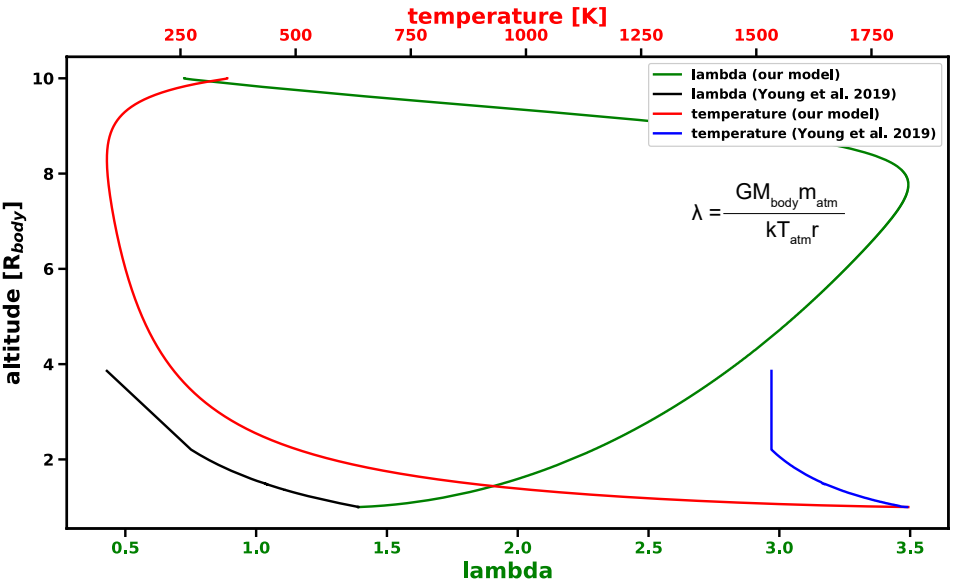

b)

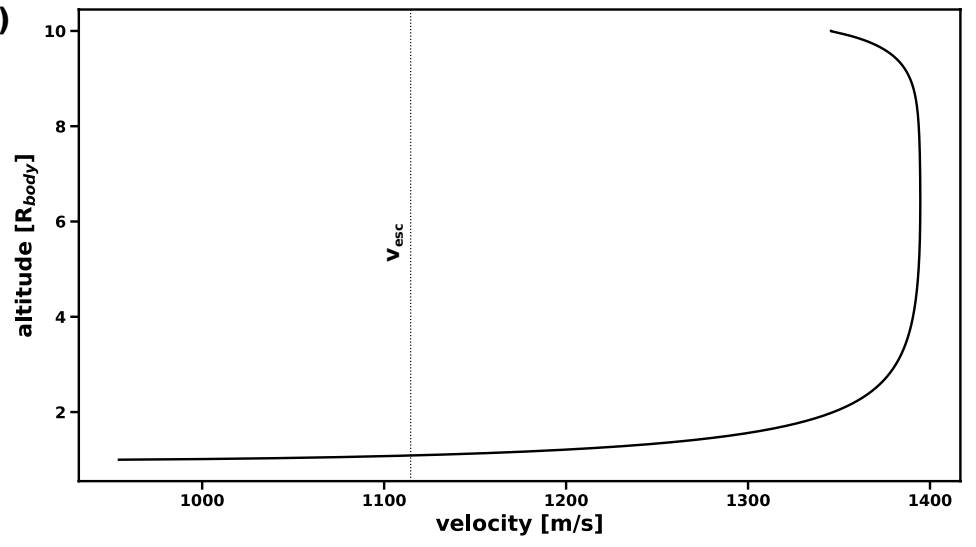

Figure 10: Another simulation run of the case described in Young et al. (2019). Here, we used the same 1D hydrodynamic atmosphere model as already described in e.g. Erkaev et al. (2015) and Erkaev et al. (2016). Panel a) shows the change of $\lambda$ (green) and temperature (red) with altitude. The Jeans escape parameter $\lambda$ is always below 6; close to the surface and in the thermosphere, $\lambda$ is clearly below the pure hydrodynamic value of $\lambda=2-3$. Clearly visible is the EUV-heating in the thermosphere as can be recognized by the clearly visible temperature inversion and accompanying decrease of $\lambda$. The blue and black lines correspond to the temperature and $\lambda$ profile of the assumed hydrostatic atmosphere of Young et al. (2019). b) Bulk flow velocity of the hydrodynamically escaping silicate atmosphere. 


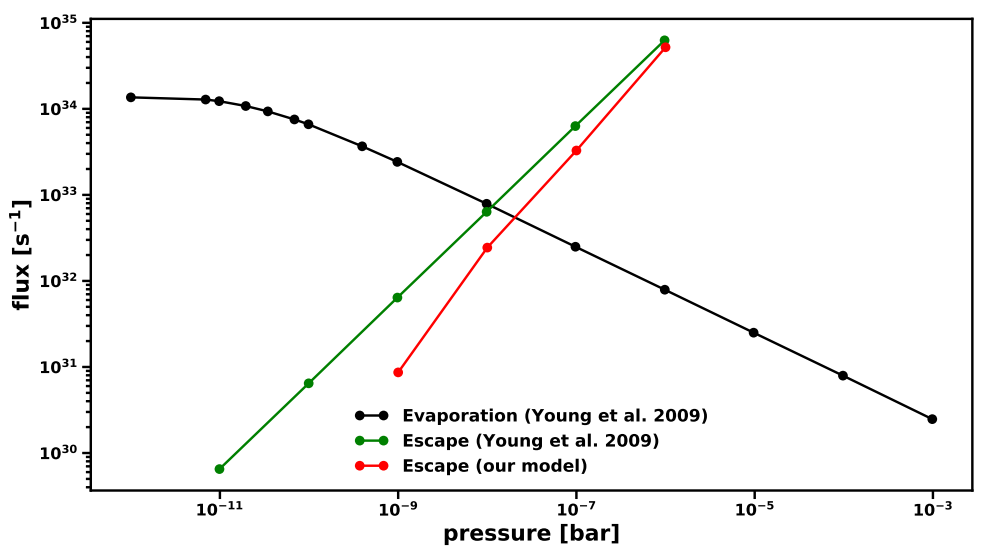

Figure 11: Outgassing from the magma ocean of the same planetary embryo (black) for different surface pressures as calculated by Young et al. (2019) and escape flux from the body as calculated by Young et al. (2019) (green) and simulated via the $1 \mathrm{D}$ hydrodynamic atmosphere model (green). In our case equilibrium between outgassing and escape is reached at a pressure of $\approx 2 \times 10^{-8}$ bar and a hydrodynamic escape flux of $\approx 5.5 \times 10^{32} \mathrm{~s}^{-1}$.

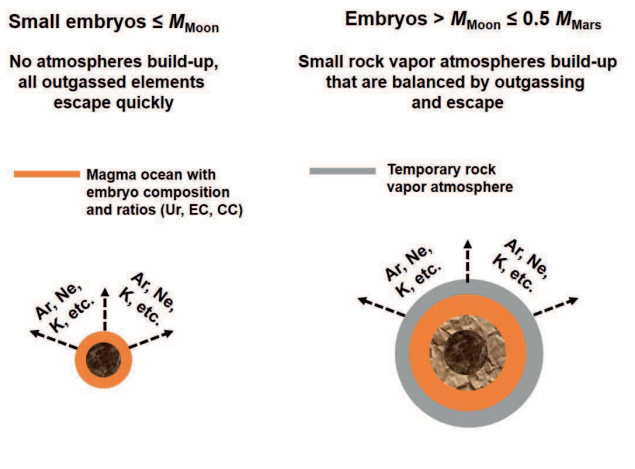

All embryos deplete in volatile elements, but isotopes are not fractionated

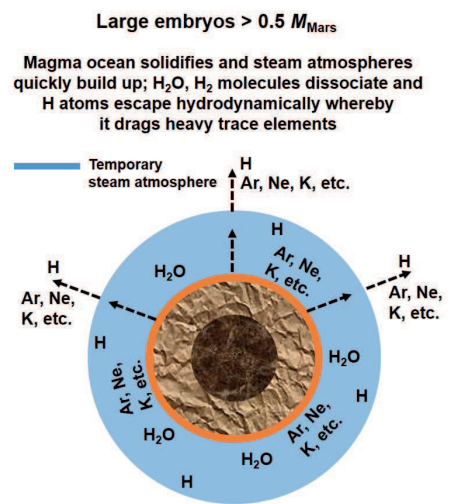

Figure 12: Illustration of the different escape regimes for small $\left(M_{\mathrm{Emb}} \leq M_{\mathrm{Moon}}\right)$, intermediate $\left.\left(M_{\text {Moon }}\right)<M_{\text {Emb }} \leq 0.5 M_{\text {Mars }}\right)$ and larger planetary embryos $\left(M_{\mathrm{Emb}} \geq 0.5 M_{\mathrm{Mars}}\right)$. 


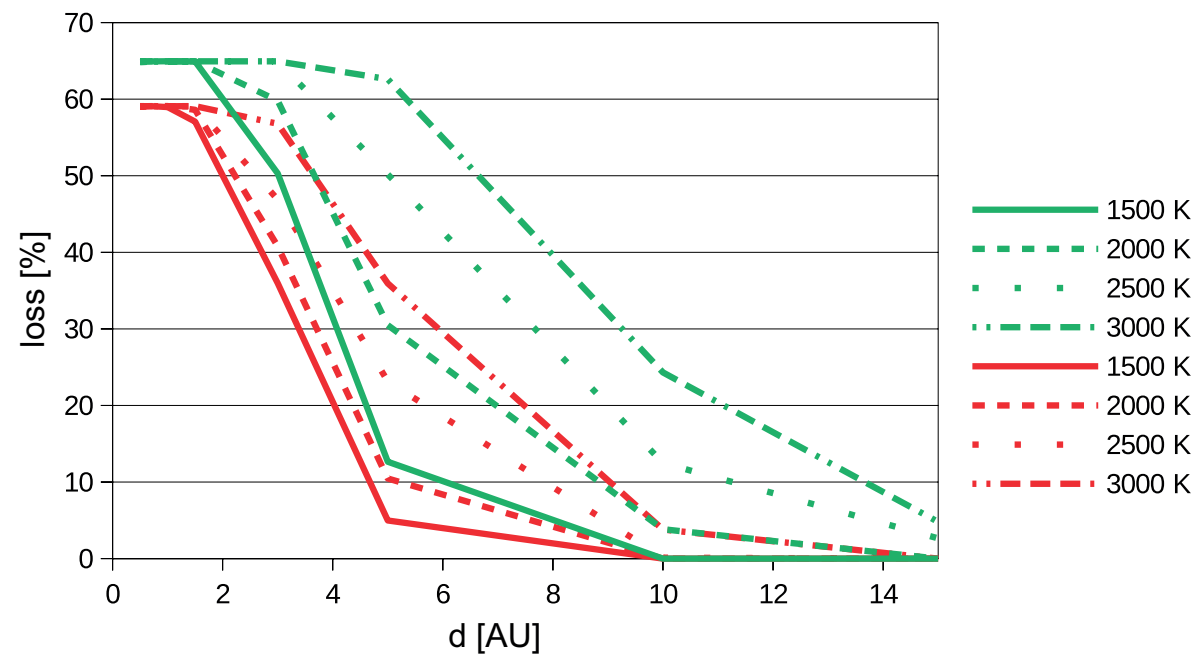

Figure 13: Relation between the orbital distance of the planetary embryo and the amount of loss for $\mathrm{K}$ around a slow rotator. The higher the orbital distance gets, however, the faster will a steam atmosphere condense if no shallow magma ocean remains at the surface; The values presented here are therefore maximum losses in case that the steam atmosphere doesn't condense. Green: $M_{\mathrm{Emb}}=1.0 M_{\mathrm{Mars}}$ and smaller; red: $M_{\mathrm{Emb}}=1.5 M_{\mathrm{Mars}}$. 